\title{
The existence and approximation of the periodic solutions for system of second order nonlinear differential equationsby using Lebesgue integrable
}

\author{
R.N.Butris \\ Merna Adel Aziz \\ College of Education College of Computer Science \& Math. \\ Department of mathematics
}

Received

08/10/2006
Accepted

05/12/2006

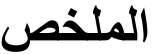

يتضمن هذا البحث درلسة وجود وقارب الح لـ ال دوري لظ لمهم ـن المع ادلات

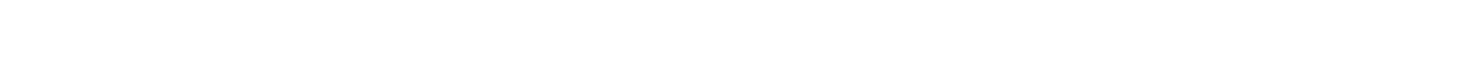
ك لـ من الدالتي ن قابلتان للتكلم لـ الليبيكي وذلك بلستخدلم الطريقة التحليلي ـة ـ العددية لدربلسة الحلول الدورية لمعادلات الفاضلية الاعتيادية اللانطية لـ ـ ـ Samoilenko.

\begin{abstract}
In this paper we study the existence and approximation of the periodicsolutions for system of second order nonlinear differential equations according to Lebesgue integrable concept and by assuming that each of measurable at $\mathrm{t}$ and bounded by $f(t, x, y, \dot{x}, \dot{y}), g(t, x, y, \dot{x}, \dot{y})$ the functions Lebesgue integrable functions numerical -- analytic method has been used to study the periodic The solutions of ordinary differential equations which were introduced by A.M. Samoilenko.
\end{abstract}




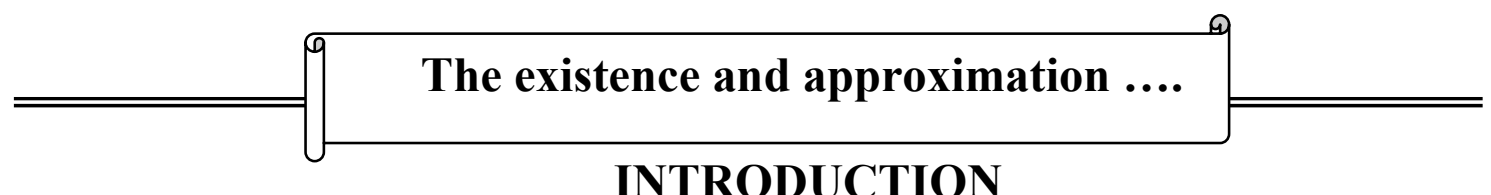

INTRODUCTION

There are many subjects in physics and technology use mathematical methods that depends on the nonlinear differential equations, and it became clear that the existence of the periodic solutions and its algorithm structure from more important problems in the present time, because of the great possibility for employment the electronic computers the numerical analytic method [6] which suggested by Samoilenko to study the periodic solutions for the linear and nonlinear differential equations became the effective mean to find the periodic solutions and its algorithm structure and this method include uniformly sequences of the periodic functions and the result of that study is the using of the periodic solutions on a wide rang in the difference of the new processes in industry and technology as in the studies $[1,2,4,5]$.

We study in this paper the system of the non linear differential equations with the form

$\left.\begin{array}{l}\frac{d^{2} x(t)}{d t^{2}}=(A+B(t))(x(t)+\dot{x}(t))+f(t, x, y, \dot{x}, \dot{y}) \\ \frac{d^{2} y(t)}{d t^{2}}=(C+D(t))(y(t)+\dot{y}(t))+g(t, x, y, \dot{x}, \dot{y})\end{array}\right\}$

represents a closed domain and bounded, the each of $x \in D \subseteq R^{n}, D$ where

the functions

$$
\begin{aligned}
& f(t, x, y, \dot{x}, \dot{y})=\left(f_{1}(t, x, y, \dot{x}, \dot{y}), f_{2}(t, x, y, \dot{x}, \dot{y}), \ldots \ldots ., f_{n}(t, x, y, \dot{x}, \dot{y})\right) \\
& g(t, x, y, \dot{x}, \dot{y})=\left(g_{1}(t, x, y, \dot{x}, \dot{y}), g_{2}(t, x, y, \dot{x}, \dot{y}), \ldots \ldots ., g_{n}(t, x, y, \dot{x}, \dot{y})\right) \\
& \text { are defined, vector and continuous in the domain } \\
& (t, x, y, \dot{x}, \dot{y}) \in[0, T] \times D \times D_{1} \times D_{2} \times D_{3}
\end{aligned}
$$

and periodic in $\mathrm{t}$ of period $\mathrm{T}$ where $D_{1}, D_{2}, D_{3}$ represents a closed domain and bounded partly from the $R^{n}$. Euclidean space and by assumption that the two functions $f(t, x, y, \dot{x}, \dot{y}), g(t, x, y, \dot{x}, \dot{y})$

satisfy the following inequalities:

$$
\begin{aligned}
& \|f(t, x, y, \dot{x}, \dot{y})\| \leq m_{1}(t) \quad, \quad\|g(t, x, y, \dot{x}, \dot{y})\| \leq m_{2}(t) \\
& \left\|f\left(t, x_{1}, y_{1}, \dot{x}_{1}, \dot{y}_{1}\right)-f\left(t, x_{2}, y_{2}, \dot{x}_{2}, \dot{y}_{2}\right)\right\| \leq K_{1}(t)\left\|x_{1}-x_{2}\right\|+K_{2}(t)\left\|y_{1}-y_{2}\right\| \\
& +K_{3}(t)\left\|\dot{x}_{1}-\dot{x}_{2}\right\|+K_{4}(t)\left\|\dot{y}_{1}-\dot{y}_{2}\right\| \\
& \left\|g\left(t, x_{1}, y_{1}, \dot{x}_{1}, \dot{y}_{1}\right)-g\left(t, x_{2}, y_{2}, \dot{x}_{2}, \dot{y}_{2}\right)\right\| \leq L_{1}(t)\left\|x_{1}-x_{2}\right\|+L_{2}(t)\left\|y_{1}-y_{2}\right\| \\
& +L_{3}(t)\left\|\dot{x}_{1}-\dot{x}_{2}\right\|+L_{4}(t)\left\|\dot{y}_{1}-\dot{y}_{2}\right\|
\end{aligned}
$$

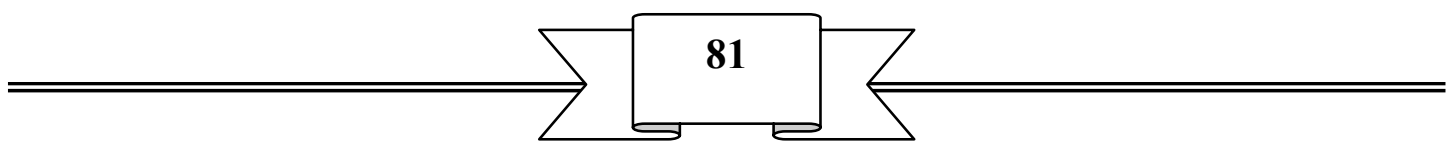




\section{R.N.Butris \& Merna Adel Aziz}

for all $t \in[0, T], x, x_{1}, x_{2} \in D, y, y_{1}, y_{2} \in D_{1}, \dot{x}, \dot{x}_{1}, \dot{x}_{2} \in D_{2}$ and $\dot{y}, \dot{y}_{1}, \dot{y}_{2} \in D_{3}$ where each of

$$
m_{1}(t), m_{2}(t), K_{1}(t), K_{2}(t), K_{3}(t), K_{4}(t), L_{1}(t), L_{2}(t), L_{3}(t), L_{4}(t)
$$

. $0 \leq t \leq T$ be Lebesgue integrable functions in the interval and by assuming that, $A=\left[A_{i j}\right], B(t)=\left[B_{i j}(t)\right], C=\left[C_{i j}\right], D(t)=\left[D_{i j}(t)\right]$ positive matrices are defined in the domain $-\infty,<0 \leq t \leq T<\infty$ continuous and periodic in thave size $(n \times n)$ and satisfy the following inequalities

$$
\begin{array}{ll}
\left\|e^{A(t-s)}\right\| \leq Q, & \left\|e^{C(t-s)}\right\| \leq R \\
\|B(t)\| \leq H \quad, & \|D(t)\| \leq J \quad,\|A\|=N_{1},\|C\|=N_{2} \\
\left\|x_{\circ}\right\|=\delta_{\circ} \quad, & \left\|y_{\circ}\right\|=\sigma_{\circ}
\end{array}
$$

where $-\infty,<0 \leq t \leq T<\infty, Q, R, H, J, \delta_{\circ}, \sigma_{\circ}$ positive constants and let $0 \leq t \leq b_{1} \leq T, 0 \leq t \leq b_{2} \leq T$ where $b_{1}, b_{2}$ are two chosen points so that

$$
\begin{aligned}
& \int_{0}^{b_{1}} m_{1}(t) d t \leq c_{1}, \int_{0}^{b_{1}} K_{1}(t) d t \leq c_{2} \leq 1, \int_{0}^{b_{1}} K_{2}(t) d t \leq c_{3} \leq 1 \\
& , \int_{0}^{b_{1}} K_{3}(t) d t \leq c_{4} \leq 1, \int_{0}^{b_{1}} K_{4}(t) d t \leq c_{5} \leq 1 \\
& \int_{0}^{b_{2}} m_{2}(t) d t \leq \delta_{1}, \int_{0}^{b_{2}} L_{1}(t) d t \leq \delta_{2} \leq 1, \int_{0}^{b_{2}} L_{2}(t) d t \leq \delta_{3} \leq 1 \\
& , \int_{0}^{b_{2}} L_{3}(t) d t \leq \delta_{4} \leq 1 \quad, \int_{0}^{b_{2}} L_{4}(t) d t \leq \delta_{5} \leq 1
\end{aligned}
$$

\section{Definition 1 [6] :-}

The system of nonlinear differential equations (1) where the righthand side defined and continuous and periodic in $t$ of period $\mathrm{T}$ in the domain (2) is said to be system - $\mathrm{T}$ if

1- The two sets $D_{f}, D_{1 f}, D_{g}, D_{1 g}$ are not empty

$$
\left.\begin{array}{l}
D_{f}=D-\frac{T}{2}\left(\frac{T}{2} N+Q C_{1}\right) \neq \phi \\
D_{1 f}=D_{2}-\left(\frac{T}{2} N+Q C_{1}\right) \neq \phi \\
D_{g}=D_{1}-\frac{T}{2}\left(\frac{T}{2} N^{*}+R \delta_{1}\right) \neq \phi \\
D_{1 g}=D_{3}-\left(\frac{T}{2} N^{*}+R \delta_{1}\right) \neq \phi
\end{array}\right\}
$$


where $N^{*}=R^{2} J \sigma_{\circ}, N=Q^{2} H \delta_{\circ},\|\|=.\max _{t \in[0 . T]} \|_{\|}$

2- The greatest eigen value of the matrix

$$
\Omega_{2}=\left[\begin{array}{cccc}
\frac{T}{2}\left(\frac{T}{2} Q\left(N_{1}+H\right)+Q C_{2}\right) & \frac{T}{2}\left(\frac{T}{2} Q H+Q C_{4}\right) & \frac{T}{2} Q C_{3} & \frac{T}{2} Q C_{5} \\
\left(\frac{T}{2} Q\left(N_{1}+H\right)+Q C_{2}\right) & \left(\frac{T}{2} Q H+Q C_{4}\right) & Q C_{3} & Q C_{5} \\
\frac{T}{2} R \delta_{2} & \frac{T}{2} R \delta_{4} & \frac{T}{2}\left(\frac{T}{2} R\left(N_{2}+J\right)+R \delta_{3}\right) & \frac{T}{2}\left(\frac{T}{2} R J+R \delta_{5}\right) \\
R \delta_{2} & R \delta_{4} & \left(\frac{T}{2} R\left(N_{2}+J\right)+R \delta_{3}\right) & \left(\frac{T}{2} R J+R \delta_{5}\right)
\end{array}\right] \text { is }
$$

not greater than 1 that is

$\lambda_{\max }\left(\Omega_{2}\right)=\frac{\xi_{1}+\sqrt{\xi_{1}^{2}+4 \xi_{2}}}{2}<1$

Where

$$
\begin{aligned}
& \xi_{1}=\frac{T}{2} \rho_{1}+\rho_{2}+\frac{T}{2} \rho_{3}+\rho_{4} \quad, \quad \xi_{2}=\frac{T^{2}}{4} v_{1} v_{3}-\frac{T^{2}}{4} \rho_{1} \rho_{3}-\frac{T}{2} \rho_{1} \rho_{4}-\frac{T}{2} \rho_{2} \rho_{3}- \\
& +\frac{T}{2} v_{1} v_{4}+\frac{T}{2} v_{2} v_{3}-\rho_{2} \rho_{4}+v_{2} v_{4} \\
& \rho_{1}=\frac{T}{2} Q\left(N_{1}+H\right)+Q C_{2}, \rho_{2}=\frac{T}{2} Q H+Q C_{4}, \rho_{3}=\frac{T}{2} R\left(N_{2}+J\right)+R \delta_{3}, \\
& \rho_{4}=\frac{T}{2} R J+R \delta_{5}, v_{1}=Q C_{3} \quad, \quad v_{2}=Q C_{5} \quad, \quad v_{3}=R \delta_{2}, v_{4}=R \delta_{4}
\end{aligned}
$$

\section{Definition 2 [6]:-}

The value of intermediary $\mu^{*}=\left(\mu_{1}^{*}, \mu_{2}^{*}\right)$ in the point $\left(t, x_{\circ}, y_{\circ}\right)$ which be on it the solution of the system

$$
\left.\begin{array}{l}
\frac{d^{2} x(t)}{d t^{2}}=(A+B(t))(x(t)+\dot{x}(t))+f(t, x, y, \dot{x}, \dot{y})-\mu_{1}^{*} \\
\frac{d^{2} y(t)}{d t^{2}}=(C+D(t))(y(t)+\dot{y}(t))+g(t, x, y, \dot{x}, \dot{y})-\mu_{2}^{*}
\end{array}\right\}
$$

Periodic in $\mathrm{t}$ of period $\mathrm{T}$ is called constant $-\Delta^{*}$ for the system (1) through the point $t=0, x=x_{\circ}, y=y_{\circ}$ if the intermediary is unique in that point . 


\section{R.N.Butris \& Merna Adel Aziz}

\section{Section One : The periodic approximate solution}

\section{for the system (1)}

\section{Lemma 1 :-}

Assume that each of $f(t, x, y, \dot{x}, \dot{y})$ and $g(t, x, y, \dot{x}, \dot{y})$ be vector function and continuous and defined in the interval $[0, \mathrm{~T}]$ then the inequality $\left(\begin{array}{l}\left\|M_{1}^{*}\left(t, x_{\circ}, y_{\circ}, 0,0\right)\right\| \\ \left\|M_{2}^{*}\left(t, x_{\circ}, y_{\circ}, 0,0\right)\right\|\end{array}\right) \leq\left(\begin{array}{l}\beta_{1}(t) N+Q C_{1} \\ \beta_{2}(t) N^{*}+R \delta_{1}\end{array}\right)$

holds for $0 \leq t \leq T, \beta_{1}(t) \leq \frac{T}{2}, \beta_{2}(t) \leq \frac{T}{2}$, where $N^{*}=R^{2} J \sigma_{\circ}, N=Q^{2} H \delta$ 。 $\beta_{1}(t)=\left[\frac{t\left(2 e^{\|A\|(T-t)}-e^{\|A\| T}-\|E\|\right)+T\left(e^{\|A\| T}-e^{\|A\|(T-t)}\right)}{e^{\|A\| T}-\|E\|}\right]$
$\beta_{2}(t)=\left[\frac{t\left(2 e^{\|C\|(T-t)}-e^{\|C\| T}-\|E\|\right)+T\left(e^{\|C\| T}-e^{\|C\|(T-t)}\right)}{e^{\|C\| T}-\|E\|}\right]$ $M_{1}^{*}\left(t, x_{\circ}, y_{\circ}, 0,0\right)=\int_{0}^{t} e^{A(t-s)}\left[B(s)\left(x_{\circ} e^{A t}+0\right)+f\left(s, x_{\circ}, y_{\circ}, 0,0\right)-\right.$ $\left.-\frac{A}{e^{A T}-E} \int_{0}^{T} e^{A(T-s)}\left[B(s)\left(x_{\circ} e^{A t}+0\right)+f\left(s, x_{\circ}, y_{\circ}, 0,0\right)\right] d s\right] d s$ $M_{2}^{*}\left(t, x_{\circ}, y_{\circ}, 0,0\right)=\int_{0}^{t} e^{C(t-s)}\left[D(s)\left(y_{\circ} e^{C t}+0\right)+g\left(s, x_{\circ}, y_{\circ}, 0,0\right)-\right.$ $\left.-\frac{C}{e^{C T}-E} \int_{0}^{T} e^{C(T-s)}\left[D(s)\left(y_{\circ} e^{C t}+0\right)+g\left(s, x_{\circ}, y_{\circ}, 0,0\right)\right] d s\right] d s$

\section{Proof}

$$
\begin{aligned}
& \left\|M_{1}\left(t, x_{\circ}, y_{\circ}, 0,0\right)\right\| \leq \\
& \leq\left[\|E\|-\left(\frac{e^{\|A\| T}-e^{\|A\|(T-t)}}{e^{\|A\| T}-\|E\|}\right)\right] \int_{0}^{t}\left\|e^{A(t-s)}\right\|\left[\|B(s)\|\left\|x_{\circ}\right\|\left\|e^{A t}\right\|+\left\|f\left(s, x_{\circ}, y_{\circ}\right)\right\|\right] d s+ \\
& \quad+\frac{e^{\|A\| T}-e^{\|A\|(T-t)}}{e^{\mid\|A\| T}-\|E\|} \int_{t}^{T}\left\|e^{A(t-s)}\right\|\left[\|B(s)\| x_{\circ}\|\| e^{A t}\|+\| f\left(s, x_{\circ}, y_{\circ}\right) \|\right] d s \\
& =\beta_{1}(t) N+Q C_{1}
\end{aligned}
$$


and also

$$
\begin{aligned}
& \left\|M_{2}\left(t, x_{\circ}, y_{\circ}, 0,0\right)\right\| \leq \\
& \leq\left[\|E\|-\left(\frac{e^{\|C\| T}-e^{\|C\|(T-t)}}{e^{\|C\| T}-\|E\|}\right)\right] \int_{0}^{t}\left\|e^{C(t-s)}\right\|\left[\|D(s)\|\left\|y_{\circ}\right\| e^{C t}\|+\| g\left(s, x_{\circ}, y_{\circ}\right) \|\right] d s+ \\
& \quad+\frac{e^{\|C\| T}-e^{\|C\|(T-t)}}{e^{\|C\| T}-\|E\|} \int_{t}^{T}\left\|e^{C(t-s)}\right\|\left[\|D(s)\|\left\|y_{\circ}\right\|\left\|e^{C t}\right\|+\left\|g\left(s, x_{\circ}, y_{\circ}\right)\right\|\right] d s \\
& =\beta_{2}(t) N^{*}+R \delta_{1}
\end{aligned}
$$

from (15) and (16) we conclude that the inequality (14) holds for

$0 \leq t \leq T, \beta_{1}(t) \leq \frac{T}{2}, \beta_{2}(t) \leq \frac{T}{2}$

Now we define the two sequences of the functions $\left\{x_{m}\left(t, x_{\circ}, y_{\circ}\right)\right\}_{m=0}^{\infty}$, $\left\{y_{m}\left(t, x_{\circ}, y_{\circ}\right)\right\}_{m=0}^{\infty}$ on the given domain as follows

$$
\begin{aligned}
x_{m+1}\left(t, x_{\circ}, y_{\circ}\right) & =x_{\circ} e^{A t}+L^{2}\left(e ^ { A ( t - s ) } \left[A x_{m}\left(t, x_{\circ}, y_{\circ}\right)+B(t)\left(x_{m}\left(t, x_{\circ}, y_{\circ}\right)+\dot{x}_{m}\left(t, x_{\circ}, y_{\circ}\right)\right)+\right.\right. \\
& \left.+f\left(t, x_{m}\left(t, x_{\circ}, y_{\circ}\right), y_{m}\left(t, x_{\circ}, y_{\circ}\right), \dot{x}_{m}\left(t, x_{\circ}, y_{\circ}\right), \dot{y}_{m}\left(t, x_{\circ}, y_{\circ}\right)\right)\right] \ldots \ldots . .(17)
\end{aligned}
$$

with $x_{\circ}\left(t, x_{\circ}, y_{\circ}\right)=x_{\circ} e^{A t} \quad, m=0,1,2$

$$
\begin{aligned}
y_{m+1}\left(t, x_{\circ}, y_{\circ}\right)= & y_{\circ} e^{C t}+L^{2}\left(e ^ { C ( t - s ) } \left[C y_{m}\left(t, x_{\circ}, y_{\circ}\right)+D(t)\left(y_{m}\left(t, x_{\circ}, y_{\circ}\right)+\dot{y}_{m}\left(t, x_{\circ}, y_{\circ}\right)\right)+\right.\right. \\
& \left.+g\left(t, x_{m}\left(t, x_{\circ}, y_{\circ}\right), y_{m}\left(t, x_{\circ}, y_{\circ}\right), \dot{x}_{m}\left(t, x_{\circ}, y_{\circ}\right), \dot{y}_{m}\left(t, x_{\circ}, y_{\circ}\right)\right)\right] \ldots \ldots . .(18)
\end{aligned}
$$

with $y_{\circ}\left(t, x_{\circ}, y_{\circ}\right)=y_{\circ} e^{C t} \quad, m=0,1,2 \ldots \ldots \ldots$

also now we define the two sequences of the functions $\left\{\dot{x}_{m}\left(t, x_{\circ}, y_{\circ}\right)\right\}_{m=0}^{\infty}$, $\left\{\dot{y}_{m}\left(t, x_{\circ}, y_{\circ}\right)\right\}_{m=0}^{\infty}$ on the given domain as follows

$$
\begin{aligned}
\dot{x}_{m+1}( & \left.t, x_{\circ}, y_{\circ}\right)=\int_{0}^{t} e^{A(t-s)}\left[A x_{m}\left(s, x_{\circ}, y_{\circ}\right)+B(s)\left(x_{m}\left(s, x_{\circ}, y_{\circ}\right)+\dot{x}_{m}\left(s, x_{\circ}, y_{\circ}\right)\right)+\right. \\
& +f\left(s, x_{m}\left(s, x_{\circ}, y_{\circ}\right), y_{m}\left(s, x_{\circ}, y_{\circ}\right), \dot{x}_{m}\left(s, x_{\circ}, y_{\circ}\right), \dot{y}_{m}\left(s, x_{\circ}, y_{\circ}\right)\right)- \\
& -\frac{A}{e^{A T}-E} \int_{0}^{T} e^{A(T-s)}\left[A x_{m}\left(s, x_{\circ}, y_{\circ}\right)+B(s)\left(x_{m}\left(s, x_{\circ}, y_{\circ}\right)+\dot{x}_{m}\left(s, x_{\circ}, y_{\circ}\right)\right)+\right. \\
& \left.\left.+f\left(s, x_{m}\left(s, x_{\circ}, y_{\circ}\right), y_{m}\left(s, x_{\circ}, y_{\circ}\right), \dot{x}_{m}\left(s, x_{\circ}, y_{\circ}\right), \dot{y}_{m}\left(s, x_{\circ}, y_{\circ}\right)\right)\right] d s\right] d s \ldots \ldots . .(19)
\end{aligned}
$$

$$
\begin{aligned}
\dot{y}_{m+1}\left(t, X_{\circ}, y_{\circ}\right)=\int_{0}^{t} e^{C(t-s)}\left[C y_{m}\left(s, x_{\circ}, y_{\circ}\right)+D(s)\left(y_{m}\left(s, X_{\circ}, y_{\circ}\right)+\dot{y}_{m}\left(s, x_{\circ}, y_{\circ}\right)\right)+\right. \\
\quad+g\left(s, x_{m}\left(s, x_{\circ}, y_{\circ}\right), y_{m}\left(s, X_{\circ}, y_{\circ}\right), \dot{x}_{m}\left(s, X_{\circ}, y_{\circ}\right), \dot{y}_{m}\left(s, x_{\circ}, y_{\circ}\right)\right) \\
\quad-\frac{C}{e^{C T}-E} \int_{0}^{T} e^{C(T-s)}\left[C y_{m}\left(s, x_{\circ}, y_{\circ}\right)+D(s)\left(y_{m}\left(s, x_{\circ}, y_{\circ}\right)+\dot{y}_{m}\left(s, x_{\circ}, y_{\circ}\right)\right)+\right. \\
\left.\left.\quad+g\left(s, x_{m}\left(s, x_{\circ}, y_{\circ}\right), y_{m}\left(s, x_{\circ}, y_{\circ}\right), \dot{x}_{m}\left(s, X_{\circ}, y_{\circ}\right), \dot{y}_{m}\left(s, x_{\circ}, y_{\circ}\right)\right)\right] d s\right] d s \ldots \ldots . .(20)
\end{aligned}
$$

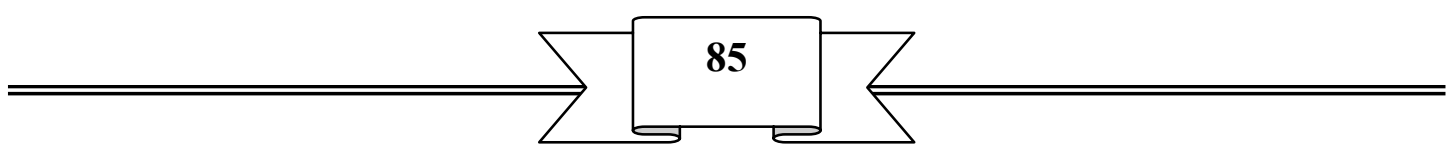




\section{R.N.Butris \& Merna Adel Aziz}

We define the two operators $\mathrm{L}, \mathrm{L}^{2}$ as follows :

$L\left(e^{A(t-s)} H\left(t, x_{\circ}, y_{\circ}\right)\right)=\int_{0}^{t} e^{A(t-s)}\left[H\left(s, x_{\circ}, y_{\circ}\right)-\Delta_{1}\right] d s \quad$,

$L\left(e^{C(t-s)} Q\left(t, x_{\circ}, y_{\circ}\right)\right)=\int_{0}^{t} e^{C(t-s)}\left[Q\left(s, x_{\circ}, y_{\circ}\right)-\Delta_{2}\right] d s$

$L^{2}\left(e^{A(t-s)} H\left(t, x_{\circ}, y_{\circ}\right)\right)=\int_{0}^{t}\left[L\left(e^{A(t-s)} H\left(s, x_{\circ}, y_{\circ}\right)\right)-\frac{1}{T} \int_{0}^{T} L\left(e^{A(t-s)} H\left(s, x_{\circ}, y_{\circ}\right)\right) d s\right] d s$

$L^{2}\left(e^{C(t-s)} Q\left(t, x_{\circ}, y_{\circ}\right)\right)=\int_{0}^{t}\left[L\left(e^{C(t-s)} Q\left(s, x_{\circ}, y_{\circ}\right)\right)-\frac{1}{T} \int_{0}^{T} L\left(e^{C(t-s)} Q\left(s, x_{\circ}, y_{\circ}\right)\right) d s\right] d s \quad$,

where

$$
\begin{aligned}
\Delta_{1}\left(x_{\circ}, y_{\circ}\right)= & \frac{A}{e^{A T}-E} \int_{0}^{T} e^{A(T-t)}\left[A x\left(t, x_{\circ}, y_{\circ}\right)+B(t)\left(x\left(t, x_{\circ}, y_{\circ}\right)+\dot{x}\left(t, x_{\circ}, y_{\circ}\right)\right)+\right. \\
& \left.+f\left(t, x\left(t, x_{\circ}, y_{\circ}\right), y\left(t, x_{\circ}, y_{\circ}\right), \dot{x}\left(t, x_{\circ}, y_{\circ}\right), \dot{y}\left(t, x_{\circ}, y_{\circ}\right)\right)\right] d t \\
H\left(t, x_{\circ}, y_{\circ}\right)= & A x\left(t, x_{\circ}, y_{\circ}\right)+B(t)\left(x\left(t, x_{\circ}, y_{\circ}\right)+\dot{x}\left(t, x_{\circ}, y_{\circ}\right)\right)+ \\
& +f\left(t, x\left(t, x_{\circ}, y_{\circ}\right), y\left(t, x_{\circ}, y_{\circ}\right), \dot{x}\left(t, x_{\circ}, y_{\circ}\right), \dot{y}\left(t, x_{\circ}, y_{\circ}\right)\right) \\
\Delta_{2}\left(x_{\circ}, y_{\circ}\right)= & \frac{C}{e^{C T}-E} \int_{0}^{T} e^{C(T-t)}\left[C y\left(t, x_{\circ}, y_{\circ}\right)+D(t)\left(y\left(t, x_{\circ}, y_{\circ}\right)+\dot{y}\left(t, x_{\circ}, y_{\circ}\right)\right)+\right. \\
+ & \left.g\left(t, x\left(t, x_{\circ}, y_{\circ}\right), y\left(t, x_{\circ}, y_{\circ}\right), \dot{x}\left(t, x_{\circ}, y_{\circ}\right), \dot{y}\left(t, x_{\circ}, y_{\circ}\right)\right)\right] d t \\
Q\left(t, x_{\circ}, y_{\circ}\right)= & C y\left(t, x_{\circ}, y_{\circ}\right)+D(t)\left(y\left(t, x_{\circ}, y_{\circ}\right)+\dot{y}\left(t, x_{\circ}, y_{\circ}\right)\right)+ \\
& +g\left(t, x\left(t, x_{\circ}, y_{\circ}\right), y\left(t, x_{\circ}, y_{\circ}\right), \dot{x}\left(t, x_{\circ}, y_{\circ}\right), \dot{y}\left(t, x_{\circ}, y_{\circ}\right)\right)
\end{aligned}
$$

It is clear that if each of $e^{A(t-s)} H\left(t, x_{\circ}, y_{\circ}\right), e^{C(t-s)} Q\left(t, x_{\circ}, y_{\circ}\right)$ be defined, Continuous and periodic in $t$ on the interval $[0, T]$ then each of $L\left(e^{A(t-s)} H\left(t, x_{\circ}, y_{\circ}\right)\right), L^{2}\left(e^{A(t-s)} H\left(t, x_{\circ}, y_{\circ}\right)\right)$ and $L\left(e^{C(t-s)} Q\left(t, x_{\circ}, y_{\circ}\right)\right)$, $L^{2}\left(e^{C(t-s)} Q\left(t, x_{\circ}, y_{\circ}\right)\right)$ are also continuous and periodic in $t$ defined on the same interval By lemma 1 we obtain

$$
\begin{aligned}
\left\|L\left(e^{A(t-s)} H_{\circ}\left(t, x_{\circ}, y_{\circ}\right)\right)\right\| \leq & \beta_{1}(t)\left\|e^{A(t-s)}\right\|\|B(t)\|\left\|x_{\circ}\right\| e^{A t}\|+\| e^{A(t-s)}\left\|\int_{0}^{t}\right\| f\left(s, x_{\circ}, y_{\circ}, 0,0\right) \| \\
& \leq \frac{T}{2} N+Q C_{1} \\
\left\|L\left(e^{C(t-s)} Q_{\circ}\left(t, x_{\circ}, y_{\circ}\right)\right)\right\| \leq & \beta_{2}(t)\left\|e^{C(t-s)}\right\| D(t)\left\|y_{\circ}\right\|\left\|e^{C t}\right\|+\left\|e^{C(t-s)}\right\| \int_{0}^{t}\left\|g\left(s, x_{\circ}, y_{\circ}, 0,0\right)\right\| \\
& \leq \frac{T}{2} N^{*}+R \delta_{1}
\end{aligned}
$$




$$
\begin{aligned}
& =\underbrace{}_{\left\|L^{2}\left(e^{A(t-s)} H_{\circ}\left(t, x_{\circ}, y_{\circ}\right)\right)\right\| \leq \alpha(t)\left\|L\left(e^{A(t-s)} H_{\circ}\left(t, x_{\circ}, y_{\circ}\right)\right)\right\|} \text { The existence and approximation .... } \\
& \leq \frac{T}{2}\left(\frac{T}{2} N+Q C_{1}\right) \\
& \left\|L^{2}\left(e^{C(t-s)} Q_{\circ}\left(t, x_{\circ}, y_{\circ}\right)\right)\right\| \leq \alpha(t)\left\|L\left(e^{C(t-s)} Q_{\circ}\left(t, x_{\circ}, y_{\circ}\right)\right)\right\| \\
& \leq \frac{T}{2}\left(\frac{T}{2} N^{*}+R \delta_{1}\right)
\end{aligned}
$$

for all $t \in[0, T], \beta_{1}(t) \leq \frac{T}{2}, \beta_{2}(t) \leq \frac{T}{2}, \alpha(t) \leq \frac{T}{2}$ where $\alpha(t)=2 t\left(1-\frac{t}{T}\right)$

\section{Theorem 1:-}

Assume that the system (1) satisfy the inequalities (3),(4),(5) and the conditions (11), (12) and if each of $f(t, x, y, \dot{x}, \dot{y})$ and $g(t, x, y, \dot{x}, \dot{y})$ are measurable functions at $t$ in the system (1) and defined in the domain (2) and satisfy the inequalities above and if the inequalities (9),(10) satisfies also and if for the system a periodic solution $x=x\left(t, x_{\circ}, y_{\circ}\right), y=y\left(t, x_{\circ}, y_{\circ}\right)$ pass through the point $\left(t, x_{\circ}, y_{\circ}\right)$ then the two sequences of the functions

$$
\begin{aligned}
x_{m+1}\left(t, x_{\circ}, y_{\circ}\right) & =x_{\circ} e^{A t}+L^{2}\left(e ^ { A ( t - s ) } \left[A x_{m}\left(t, x_{\circ}, y_{\circ}\right)+B(t)\left(x_{m}\left(t, x_{\circ}, y_{\circ}\right)+\dot{x}_{m}\left(t, x_{\circ}, y_{\circ}\right)\right)+\right.\right. \\
+ & \left.\left.f\left(t, x_{m}\left(t, x_{\circ}, y_{\circ}\right), y_{m}\left(t, x_{\circ}, y_{\circ}\right), \dot{x}_{m}\left(t, x_{\circ}, y_{\circ}\right), \dot{y}_{m}\left(t, x_{\circ}, y_{\circ}\right)\right)\right]\right)
\end{aligned}
$$

with $x_{\circ}\left(t, x_{\circ}, y_{\circ}\right)=x_{\circ} e^{A t} \quad, \frac{d x_{m}}{d t}=\dot{x}_{m}\left(t, x_{\circ}, y_{\circ}\right) \quad, m=0,1,2, \ldots .$.

$$
\begin{array}{r}
y_{m+1}\left(t, x_{\circ}, y_{\circ}\right)=y_{\circ} e^{C t}+L^{2}\left(e ^ { C ( t - s ) } \left[C y_{m}\left(t, x_{\circ}, y_{\circ}\right)+D(t)\left(y_{m}\left(t, x_{\circ}, y_{\circ}\right)+\dot{y}_{m}\left(t, x_{\circ}, y_{\circ}\right)\right)+\right.\right. \\
\left.\left.+g\left(t, x_{m}\left(t, x_{\circ}, y_{\circ}\right), y_{m}\left(t, x_{\circ}, y_{\circ}\right), \dot{x}_{m}\left(t, x_{\circ}, y_{\circ}\right), \dot{y}_{m}\left(t, x_{\circ}, y_{\circ}\right)\right)\right]\right)
\end{array}
$$

with $y_{\circ}\left(t, x_{\circ}, y_{\circ}\right)=y_{\circ} e^{C t} \quad, \frac{d y_{m}}{d t}=\dot{y}_{m}\left(t, x_{\circ}, y_{\circ}\right) \quad, m=0,1,2, \ldots \ldots$

periodicity in $\mathrm{t}$ of period $\mathrm{T}$, its uniformly convergent when $m \rightarrow \infty$ in the domain

$$
\left(t, x_{\circ}, y_{\circ}\right) \in[0, T] \times D_{f} \times D_{g}
$$

from the two functions $x_{\infty}\left(t, x_{\circ}, y_{\circ}\right)$ and $y_{\infty}\left(t, x_{\circ}, y_{\circ}\right)$ are defined in the domain (27) continuous and periodic in $t$ of period $\mathrm{T}$ and satisfies the system of integral equations

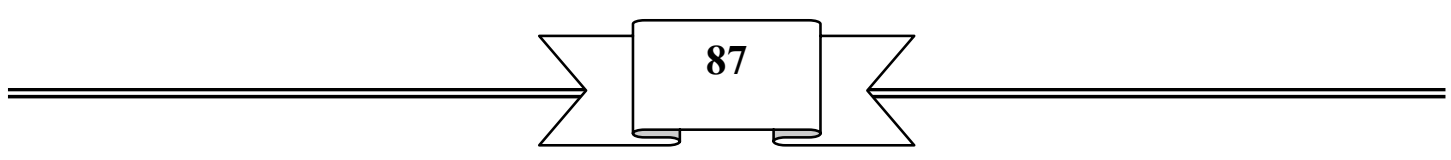




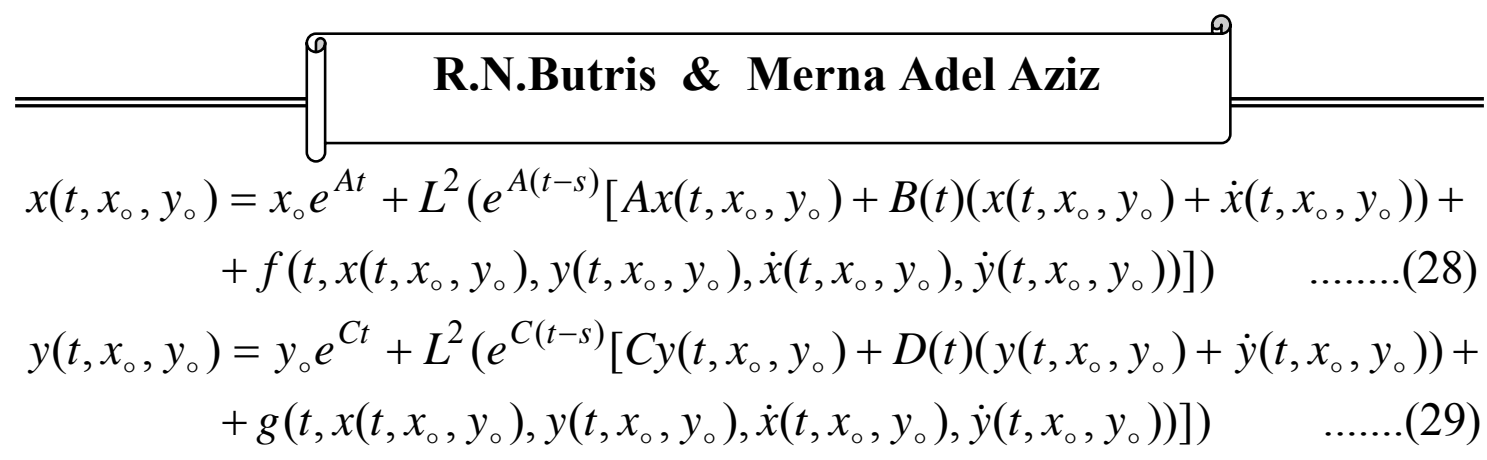

which are a unique solution for the system (1) and satisfies the following inequality

$$
\left(\begin{array}{l}
\left\|x_{\infty}\left(t, x_{\circ}, y_{\circ}\right)-x_{m}\left(t, x_{\circ}, y_{\circ}\right)\right\| \\
\left\|\dot{x}_{\infty}\left(t, x_{\circ}, y_{\circ}\right)-\dot{x}_{m}\left(t, x_{\circ}, y_{\circ}\right)\right\| \\
\left\|y_{\infty}\left(t, x_{\circ}, y_{\circ}\right)-y_{m}\left(t, x_{\circ}, y_{\circ}\right)\right\| \\
\left\|\dot{y}_{\infty}\left(t, x_{\circ}, y_{\circ}\right)-\dot{y}_{m}\left(t, x_{\circ}, y_{\circ}\right)\right\|
\end{array}\right) \leq \Omega_{2}^{m}\left(E-\Omega_{2}\right)^{-1} V_{\circ}
$$

where $V_{\circ}=\left(\begin{array}{c}\frac{T}{2}\left(\frac{T}{2} N+Q C_{1}\right) \\ \left(\frac{T}{2} N+Q C_{1}\right) \\ \frac{T}{2}\left(\frac{T}{2} N^{*}+R \delta_{1}\right) \\ \left(\frac{T}{2} N^{*}+R \delta_{1}\right)\end{array}\right), \quad$ E the unit matrix

\section{Proof :-}

When we look to the two sequences of the functions

$$
\begin{aligned}
& x_{1}\left(t, x_{\circ}, y_{\circ}\right), x_{2}\left(t, x_{\circ}, y_{\circ}\right), \ldots \ldots, x_{m}\left(t, x_{\circ}, y_{\circ}\right), \ldots \ldots . \\
& y_{1}\left(t, x_{\circ}, y_{\circ}\right), y_{2}\left(t, x_{\circ}, y_{\circ}\right), \ldots \ldots, y_{m}\left(t, x_{\circ}, y_{\circ}\right), \ldots \ldots . .
\end{aligned}
$$

that are defined in (25),(26) we find that each of the sequences of the functions are defined and continuous in the domain (2) and periodic in $t$ of period $\mathrm{T}$.

By lemma 1 and from (25) when $\mathrm{m}=0$ we obtain

$$
\left\|x_{1}\left(t, x_{\circ}, y_{\circ}\right)-x_{\circ}\left(t, x_{\circ}, y_{\circ}\right)\right\|=\left\|L^{2}\left(e^{A(t-s)} H_{\circ}\left(t, x_{\circ}, y_{\circ}\right)\right)\right\|
$$

By using (23) we get $\left\|x_{1}\left(t, x_{\circ}, y_{\circ}\right)-x_{\circ}\left(t, x_{\circ}, y_{\circ}\right)\right\| \leq \frac{T}{2}\left(\frac{T}{2} N+Q C_{1}\right)$

Then $x_{1}\left(t, x_{\circ}, y_{\circ}\right) \in D$ for all $x_{\circ} \in D_{f}, y_{\circ} \in D_{g}$

also from the relation (19) and by lemma 1 when $m=0$ we obtain $\left\|\dot{x}_{1}\left(t, x_{\circ}, y_{\circ}\right)-0\right\|=\left\|L\left(e^{A(t-s)} H_{\circ}\left(t, x_{\circ}, y_{\circ}\right)\right)\right\|$

By using (21) we get $\quad\left\|\dot{x}_{1}\left(t, x_{\circ}, y_{\circ}\right)-0\right\| \leq \frac{T}{2} N+Q C_{1}$

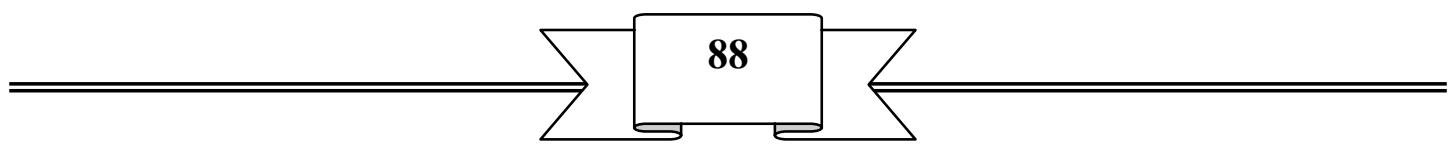




\section{The existence and approximation}

Then $\dot{x}_{1}\left(t, x_{\circ}, y_{\circ}\right) \in D$ for all $x_{\circ} \in D_{f}, y_{\circ} \in D_{g}$

also from the relation (26) when $\mathrm{m}=0$ we obtain

$\left\|y_{1}\left(t, x_{\circ}, y_{\circ}\right)-y_{\circ}\left(t, x_{\circ}, y_{\circ}\right)\right\|=\left\|L^{2}\left(e^{C(t-s)} Q_{\circ}\left(t, x_{\circ}, y_{\circ}\right)\right)\right\|$

By using (24) we get $\quad\left\|y_{1}\left(t, x_{\circ}, y_{\circ}\right)-y_{\circ}\left(t, x_{\circ}, y_{\circ}\right)\right\| \leq \frac{T}{2}\left(\frac{T}{2} N^{*}+R \delta_{1}\right)$

Then $y_{1}\left(t, x_{\circ}, y_{\circ}\right) \in D_{1}$ for all $x_{\circ} \in D_{f}, y_{\circ} \in D_{g}$

also from the relation $(20)$ when $\mathrm{m}=0$ we obtain

$\left\|\dot{y}_{1}\left(t, x_{\circ}, y_{\circ}\right)-0\right\|=\left\|L\left(e^{C(t-s)} Q_{\circ}\left(t, x_{\circ}, y_{\circ}\right)\right)\right\|$

By using (24) we get $\quad\left\|\dot{y}_{1}\left(t, x_{\circ}, y_{\circ}\right)-0\right\| \leq \frac{T}{2} N^{*}+R \delta_{1}$

Then $\dot{y}_{1}\left(t, x_{\circ}, y_{\circ}\right) \in D_{3}$ for all $x_{\circ} \in D_{f}, y_{\circ} \in D_{g}$

and by using the mathematical induction we can prove the truth of the following inequalities for $m \geq 1$

$\left.\begin{array}{c}\left\|x_{m}\left(t, x_{\circ}, y_{\circ}\right)-x_{\circ}\left(t, x_{\circ}, y_{\circ}\right)\right\| \leq \frac{T}{2}\left(\frac{T}{2} N+Q C_{1}\right) \\ \left\|\dot{x}_{m}\left(t, x_{\circ}, y_{\circ}\right)-0\right\| \leq \frac{T}{2} N+Q C_{1} \\ \left\|y_{m}\left(t, x_{\circ}, y_{\circ}\right)-y_{\circ}\left(t, x_{\circ}, y_{\circ}\right)\right\| \leq \frac{T}{2}\left(\frac{T}{2} N^{*}+R \delta_{1}\right) \\ \left\|\dot{y}_{m}\left(t, x_{\circ}, y_{\circ}\right)-0\right\| \leq \frac{T}{2} N^{*}+R \delta_{1}\end{array}\right\}$

that is

$x_{m}\left(t, x_{\circ}, y_{\circ}\right) \in D, \dot{x}_{m}\left(t, x_{\circ}, y_{\circ}\right) \in D_{2}, y_{m}\left(t, x_{\circ}, y_{\circ}\right) \in D_{1}, \dot{y}_{m}\left(t, x_{\circ}, y_{\circ}\right) \in D_{3}$

for all $x_{\circ} \in D_{f}, y_{\circ} \in D_{g}$

now we prove that the two sequences

$\left\{x_{m}\left(t, x_{\circ}, y_{\circ}\right)\right\}_{m=0}^{\infty},\left\{y_{m}\left(t, x_{\circ}, y_{\circ}\right)\right\}_{m=0}^{\infty}$ are

uniformly convergent in the domain (27) and thereupon each of the two ends for the two sequences are periodicity and continuous in the same domain .

from the previous proof we find

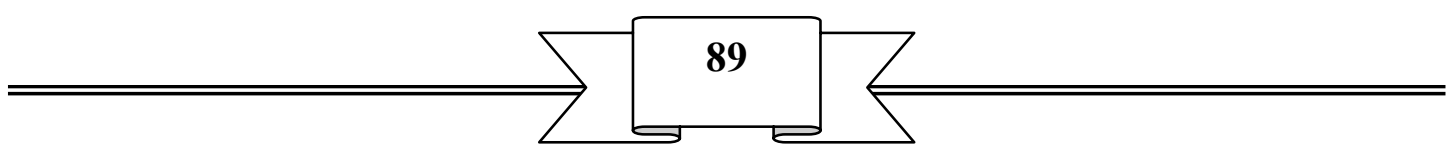




$$
\begin{aligned}
& \hline \hline x_{1}\left(t, x_{\circ}, y_{\circ}\right)-x_{\circ}\left(t, x_{\circ}, y_{\circ}\right) \| \leq \frac{T}{2}\left(\frac{T}{2} N+Q C_{1}\right), \\
& \left\|\dot{x}_{1}\left(t, x_{\circ}, y_{\circ}\right)-0\right\| \leq \frac{T}{2} N+Q C_{1}, \\
& \left\|y_{1}\left(t, x_{\circ}, y_{\circ}\right)-y_{\circ}\left(t, x_{\circ}, y_{\circ}\right)\right\| \leq \frac{T}{2}\left(\frac{T}{2} N^{*}+R \delta_{1}\right), \\
& \left\|\dot{y}_{1}\left(t, x_{\circ}, y_{\circ}\right)-0\right\| \leq \frac{T}{2} N^{*}+R \delta_{1} .
\end{aligned}
$$

Now when $\mathrm{m}=1$ in (25) and by using (23) we find

$$
\begin{gathered}
\left\|x_{2}\left(t, x_{\circ}, y_{\circ}\right)-x_{1}\left(t, x_{\circ}, y_{\circ}\right)\right\| \leq \alpha(t)\left(\beta_{1}(t) Q\left(N_{1}+H\right)+Q C_{2}\right)\left\|x_{1}\left(t, x_{\circ}, y_{\circ}\right)-x_{\circ}\left(t, x_{\circ}, y_{\circ}\right)\right\|+ \\
+\alpha(t)\left(\beta_{1}(t) Q H+Q C_{4}\right) \| \dot{x}_{1}\left(t, x_{\circ}, y_{\circ}\right)-0 \mid \\
+\alpha(t) Q C_{3}\left\|y_{1}\left(t, x_{\circ}, y_{\circ}\right)-y_{\circ}\left(t, x_{\circ}, y_{\circ}\right)\right\|+ \\
+\alpha(t) Q C_{5}\left\|\dot{y}_{1}\left(t, x_{\circ}, y_{\circ}\right)-0\right\|
\end{gathered}
$$

also when $\mathrm{m}=1$ in (19) and by using (21) we find

$$
\begin{gathered}
\left\|\dot{x}_{2}\left(t, x_{\circ}, y_{\circ}\right)-\dot{x}_{1}\left(t, x_{\circ}, y_{\circ}\right)\right\| \leq\left(\beta_{1}(t) Q\left(N_{1}+H\right)+Q C_{2}\right)\left\|x_{1}\left(t, x_{\circ}, y_{\circ}\right)-x_{\circ}\left(t, x_{\circ}, y_{\circ}\right)\right\|+ \\
+\left(\beta_{1}(t) Q H+Q C_{4}\right)\left\|\dot{x}_{1}\left(t, x_{\circ}, y_{\circ}\right)-0\right\| \\
+Q C_{3}\left\|y_{1}\left(t, x_{\circ}, y_{\circ}\right)-y_{\circ}\left(t, x_{\circ}, y_{\circ}\right)\right\|+ \\
+Q C_{5}\left\|\dot{y}_{1}\left(t, x_{\circ}, y_{\circ}\right)-0\right\|
\end{gathered}
$$

also when $\mathrm{m}=1$ in (26) and by using (24) we find

$$
\begin{aligned}
\left\|y_{2}\left(t, x_{\circ}, y_{\circ}\right)-y_{1}\left(t, x_{\circ}, y_{\circ}\right)\right\| & \leq \alpha(t) R \delta_{2}\left\|x_{1}\left(t, x_{\circ}, y_{\circ}\right)-x_{\circ}\left(t, x_{\circ}, y_{\circ}\right)\right\|+ \\
+\alpha(t) R \delta_{4}\left\|\dot{x}_{1}\left(t, x_{\circ}, y_{\circ}\right)-0\right\| & +\alpha(t)\left(\beta_{2}(t) R\left(N_{2}+J\right)+R \delta_{3}\right)\left\|y_{1}\left(t, x_{\circ}, y_{\circ}\right)-y_{\circ}\left(t, x_{\circ}, y_{\circ}\right)\right\|+ \\
& +\alpha(t)\left(\beta_{2}(t) R J+R \delta_{5}\right)\left\|\dot{y}_{1}\left(t, x_{\circ}, y_{\circ}\right)-0\right\|
\end{aligned}
$$

also when $\mathrm{m}=1$ in (22) and by using (20) we find

$$
\begin{aligned}
&\left\|\dot{y}_{2}\left(t, x_{\circ}, y_{\circ}\right)-\dot{y}_{1}\left(t, x_{\circ}, y_{\circ}\right)\right\| \leq R \delta_{2}\left\|x_{1}\left(t, x_{\circ}, y_{\circ}\right)-x_{\circ}\left(t, x_{\circ}, y_{\circ}\right)\right\|+ \\
&+R \delta_{4}\left\|\dot{x}_{1}\left(t, x_{\circ}, y_{\circ}\right)-0\right\|+\left(\beta_{2}(t) R\left(N_{2}+J\right)+R \delta_{3}\right)\left\|y_{1}\left(t, x_{\circ}, y_{\circ}\right)-y_{\circ}\left(t, x_{\circ}, y_{\circ}\right)\right\|+ \\
&+\left(\beta_{2}(t) R J+R \delta_{5}\right)\left\|\dot{y}_{1}\left(t, x_{\circ}, y_{\circ}\right)-0\right\|
\end{aligned}
$$

And so by using the mathematical induction we can prove the truth of the following two inequalities :

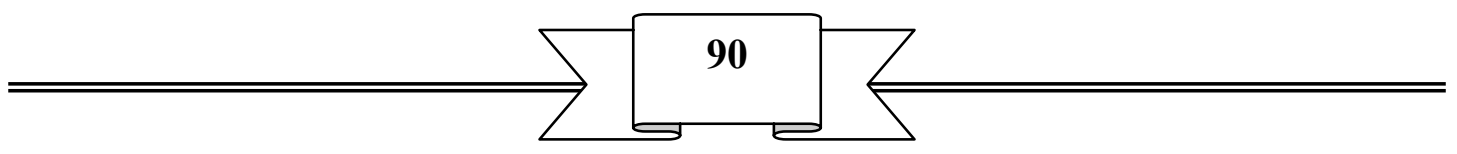




$$
\begin{aligned}
& \hline \int_{m+1}\left(t, x_{\circ}, y_{\circ}\right)-x_{m}\left(t, x_{\circ}, y_{\circ}\right) \| \leq \\
& \leq \alpha(t)\left(\beta_{1}(t) Q\left(N_{1}+H\right)+Q C_{2}\right)\left\|x_{m}\left(t, x_{\circ}, y_{\circ}\right)-x_{m-1}\left(t, x_{\circ}, y_{\circ}\right)\right\|+ \\
& +\alpha(t)\left(\beta_{1}(t) Q H+Q C_{4}\right)\left\|\dot{x}_{m}\left(t, x_{\circ}, y_{\circ}\right)-\dot{x}_{m-1}\left(t, x_{\circ}, y_{\circ}\right)\right\|+ \\
& +\alpha(t) Q C_{3}\left\|y_{m}\left(t, x_{\circ}, y_{\circ}\right)-y_{m-1}\left(t, x_{\circ}, y_{\circ}\right)\right\|+ \\
& +\alpha(t) Q C_{5}\left\|\dot{y}_{m}\left(t, x_{\circ}, y_{\circ}\right)-\dot{y}_{m-1}\left(t, x_{\circ}, y_{\circ}\right)\right\| \\
& \left\|\dot{x}_{m+1}\left(t, x_{\circ}, y_{\circ}\right)-\dot{x}_{m}\left(t, x_{\circ}, y_{\circ}\right)\right\| \leq \\
& \leq\left(\beta_{1}(t) Q\left(N_{1}+H\right)+Q C_{2}\right)\left\|x_{m}\left(t, x_{\circ}, y_{\circ}\right)-x_{m-1}\left(t, x_{\circ}, y_{\circ}\right)\right\|+ \\
& +\left(\beta_{1}(t) Q H+Q C_{4}\right)\left\|\dot{x}_{m}\left(t, x_{\circ}, y_{\circ}\right)-\dot{x}_{m-1}\left(t, x_{\circ}, y_{\circ}\right)\right\|+ \\
& +Q C_{3}\left\|y_{m}\left(t, x_{\circ}, y_{\circ}\right)-y_{m-1}\left(t, x_{\circ}, y_{\circ}\right)\right\|+ \\
& +Q C_{5}\left\|\dot{y}_{m}\left(t, x_{\circ}, y_{\circ}\right)-\dot{y}_{m-1}\left(t, x_{\circ}, y_{\circ}\right)\right\| \\
& \quad\left\|y_{m+1}\left(t, x_{\circ}, y_{\circ}\right)-y_{m}\left(t, x_{\circ}, y_{\circ}\right)\right\| \leq \\
& \leq \alpha(t) R \delta_{2}\left\|x_{m}\left(t, x_{\circ}, y_{\circ}\right)-x_{m-1}\left(t, x_{\circ}, y_{\circ}\right)\right\|+ \\
& +\alpha(t) R \delta_{4}\left\|\dot{x}_{m}\left(t, x_{\circ}, y_{\circ}\right)-\dot{x}_{m-1}\left(t, x_{\circ}, y_{\circ}\right)\right\|+ \\
& +\alpha(t)\left(\beta_{2}(t) R\left(N_{2}+J\right)+R \delta_{3}\right)\left\|y_{m}\left(t, x_{\circ}, y_{\circ}\right)-y_{m-1}\left(t, x_{\circ}, y_{\circ}\right)\right\|+ \\
& +\alpha(t)\left(\beta_{2}(t) R J+R \delta_{5}\right)\left\|\dot{y}_{m}\left(t, x_{\circ}, y_{\circ}\right)-\dot{y}_{m-1}\left(t, x_{\circ}, y_{\circ}\right)\right\| \\
& +\dot{y}_{m+1}\left(t, x_{\circ}, y_{\circ}\right)-\dot{y}_{m}\left(t, x_{\circ}, y_{\circ}\right) \| \leq \\
& \leq R \delta_{2}\left\|x_{m}\left(t, x_{\circ}, y_{\circ}\right)-x_{m-1}\left(t, x_{\circ}, y_{\circ}\right)\right\|+ \\
& +\left(\beta _ { 2 } ( t ) R \left(\dot{x}_{m}\left(t, x_{\circ}, y_{\circ}\right)-\dot{x}_{m-1}\left(t, x_{\circ}, y_{\circ}\right) \|+\right.\right.
\end{aligned}
$$

we rewrite (32),(33),(34),(35) with vectors form and on the following mode

$$
V_{m+1}(t) \leq \Omega_{1}(t) V_{m}(t)
$$

where

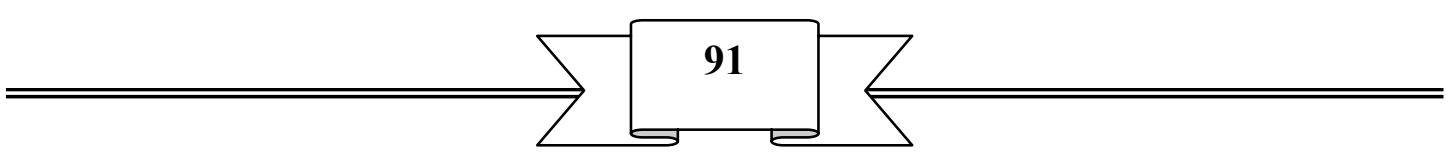




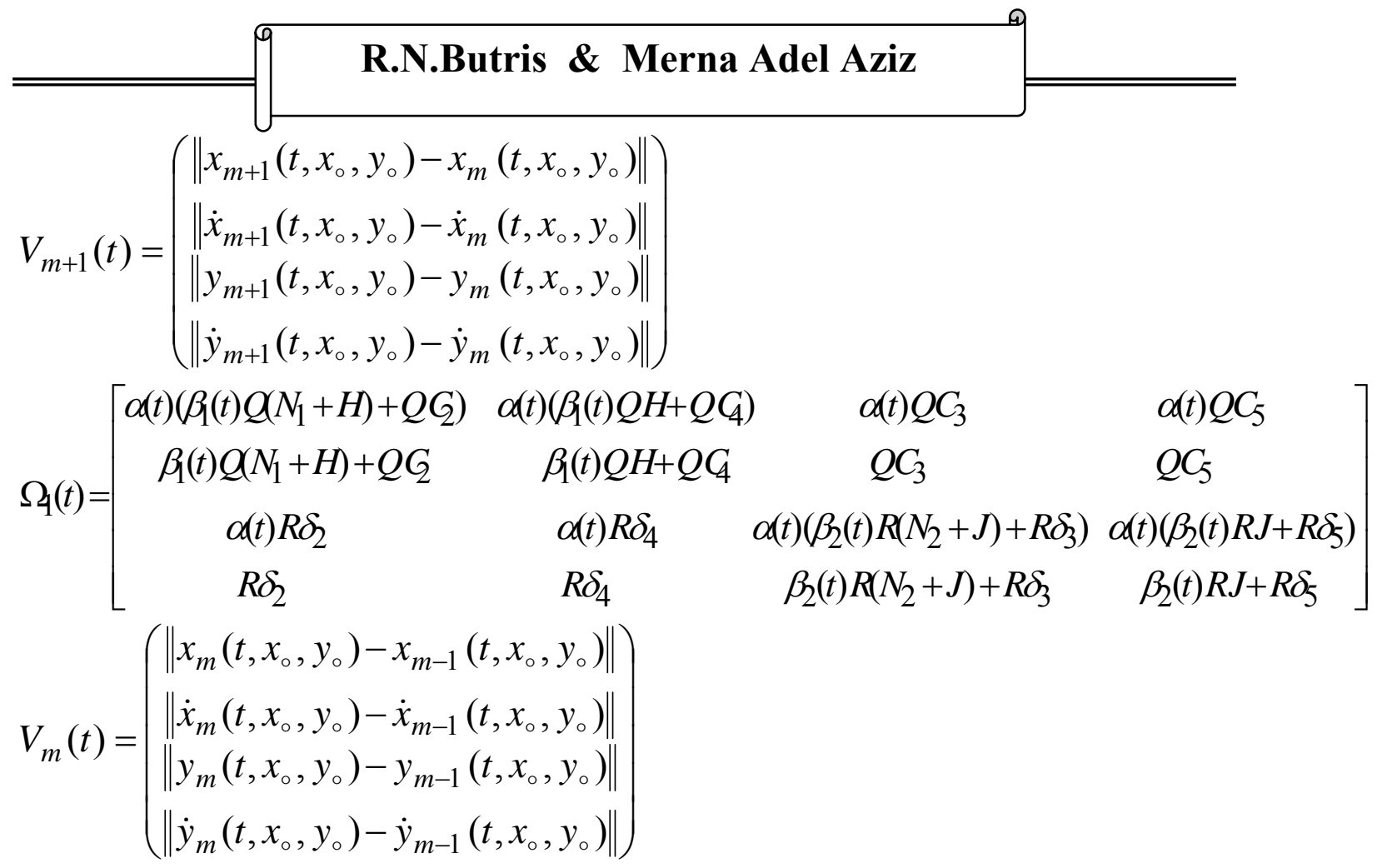

Now we take the maximum value for the two sides of the inequality (36)for

$0 \leq t \leq T$

we find that

$V_{m+1} \leq \Omega_{2} V_{m}$

where $\Omega_{2}=\max _{t \in[0, T]} \Omega_{1}(t)$

$\Omega_{2}=\left[\begin{array}{cccc}\frac{T}{2}\left(\frac{T}{2} Q\left(N_{1}+H\right)+Q G\right) & \frac{T}{2}\left(\frac{T}{2} Q H+Q C_{4}\right) & \frac{T}{2} Q C_{3} & \frac{T}{2} Q C_{5} \\ \left(\frac{T}{2} Q\left(N_{1}+H\right)+Q G\right) & \left(\frac{T}{2} Q H+Q C_{4}\right) & Q C_{3} & Q C_{5} \\ \frac{T}{2} R \delta_{2} & \frac{T}{2} R \delta_{4} & \frac{T}{2}\left(\frac{T}{2} R\left(N_{2}+J\right)+R \delta_{3}\right) & \frac{T}{2}\left(\frac{T}{2} R J+R \delta_{5}\right) \\ R \delta_{2} & R \delta_{4} & \left(\frac{T}{2} R\left(N_{2}+J\right)+R \delta_{3}\right) & \left(\frac{T}{2} R J+R \delta_{5}\right)\end{array}\right]$

and by repetition (37) we have

$V_{m+1} \leq \Omega_{2}^{m} V_{\text {。 }}$

also we find

$\sum_{i=1}^{m} V_{i} \leq \sum_{i=1}^{m} \Omega_{2}^{i-1} V_{\text {。 }}$

since the matrix $\Omega_{2}$ has the greatest eigen value as in (12) this lead to that the sequence (39) is uniformly convergent that is

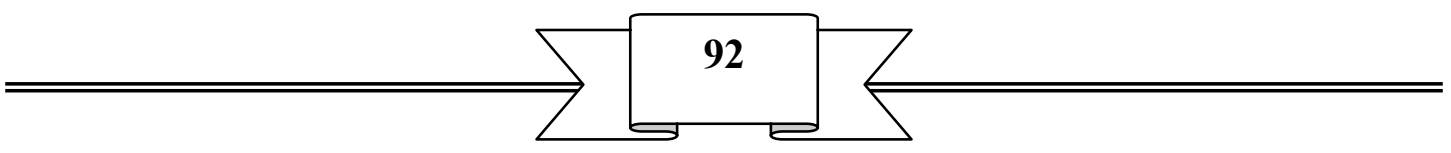




\section{The existence and approximation}

$$
\lim _{m \rightarrow \infty} \sum_{i=1}^{m} \Omega_{2}^{i-1} V_{\circ}=\sum_{i=1}^{\infty} \Omega_{2}^{i-1} V_{\circ}=\left(E-\Omega_{2}\right)^{-1} V_{\circ}
$$

and then the relation (40) ascertain on the convergence of sequences of the

functions $\left[x_{m}\left(t, x_{\circ}, y_{\circ}\right), \dot{x}_{m}\left(t, x_{\circ}, y_{\circ}\right), y_{m}\left(t, x_{\circ}, y_{\circ}\right), \dot{y}_{m}\left(t, x_{\circ}, y_{\circ}\right)\right]$ ]on the domain (27).

We assume that

$$
\left.\begin{array}{l}
\lim _{m \rightarrow \infty} x_{m}\left(t, x_{\circ}, y_{\circ}\right)=x_{\infty}\left(t, x_{\circ}, y_{\circ}\right), \\
\lim _{m \rightarrow \infty} \dot{x}_{m}\left(t, x_{\circ}, y_{\circ}\right)=\dot{x}_{\infty}\left(t, x_{\circ}, y_{\circ}\right), \\
\lim _{m \rightarrow \infty} y_{m}\left(t, x_{\circ}, y_{\circ}\right)=y_{\infty}\left(t, x_{\circ}, y_{\circ}\right), \\
\lim _{m \rightarrow \infty} \dot{y}_{m}\left(t, x_{\circ}, y_{\circ}\right)=\dot{y}_{\infty}\left(t, x_{\circ}, y_{\circ}\right),
\end{array}\right\}
$$

since each of the sequences of the functions (25),(19),(26),(20) are continuous and periodic in $t$ of period $\mathrm{T}$ then each of the ends for the sequences are continuous and periodic in $\mathrm{t}$ of period $\mathrm{T}$ and thereupon be $x_{\infty}\left(t, x_{\circ}, y_{\circ}\right)=x\left(t, x_{\circ}, y_{\circ}\right), y_{\infty}\left(t, x_{\circ}, y_{\circ}\right)=y\left(t, x_{\circ}, y_{\circ}\right)$ $\dot{x}_{\infty}\left(t, x_{\circ}, y_{\circ}\right)=\dot{x}\left(t, x_{\circ}, y_{\circ}\right), \dot{y}_{\infty}\left(t, x_{\circ}, y_{\circ}\right)=\dot{y}\left(t, x_{\circ}, y_{\circ}\right)$

And in addition for that the using of lemma 1 and the relation (40) the inequality (30) satisfies for $m \geq 0$ now we prove that $x\left(t, x_{\circ}, y_{\circ}\right), y\left(t, x_{\circ}, y_{\circ}\right)$ are unique solution for the system (1) by contradiction .

we assume there at least another two different solutions $x\left(t, x_{\circ}, y_{\circ}\right), y\left(t, x_{\circ}, y_{\circ}\right)$, $r\left(t, x_{\circ}, y_{\circ}\right), u\left(t, x_{\circ}, y_{\circ}\right)$ for the system (1) are each of it defined and continuous and periodic in $t$ of period $\mathrm{T}$. from the form in below :

$$
\begin{aligned}
r\left(t, x_{\circ}, y_{\circ}\right)=x_{\circ} e^{A t}+L^{2}\left(e ^ { A ( t - s ) } \left[A r\left(t, x_{\circ}, y_{\circ}\right)+B(t)\left(r\left(t, x_{\circ}, y_{\circ}\right)+\dot{r}\left(t, x_{\circ}, y_{\circ}\right)\right)+\right.\right. & \left.\left.+f\left(t, r\left(t, x_{\circ}, y_{\circ}\right), u\left(t, x_{\circ}, y_{\circ}\right), \dot{r}\left(t, x_{\circ}, y_{\circ}\right), \dot{u}\left(t, x_{\circ}, y_{\circ}\right)\right)\right]\right) \\
H^{*}\left(t, x_{\circ}, y_{\circ}\right)= & A r\left(t, x_{\circ}, y_{\circ}\right)+B(t)\left(r\left(t, x_{\circ}, y_{\circ}\right)+\dot{r}\left(t, x_{\circ}, y_{\circ}\right)\right)+ \\
& +f\left(t, r\left(t, x_{\circ}, y_{\circ}\right), u\left(t, x_{\circ}, y_{\circ}\right), \dot{r}\left(t, x_{\circ}, y_{\circ}\right), \dot{u}\left(t, x_{\circ}, y_{\circ}\right)\right) \ldots \ldots . .(42) \\
\dot{r}\left(t, x_{\circ}, y_{\circ}\right)= & L\left(e ^ { A ( t - s ) } \left[A r\left(t, x_{\circ}, y_{\circ}\right)+B(t)\left(r\left(t, x_{\circ}, y_{\circ}\right)+\dot{r}\left(t, x_{\circ}, y_{\circ}\right)\right)+\right.\right. \\
+ & \left.\left.f\left(t, r\left(t, x_{\circ}, y_{\circ}\right), u\left(t, x_{\circ}, y_{\circ}\right), \dot{r}\left(t, x_{\circ}, y_{\circ}\right), \dot{u}\left(t, x_{\circ}, y_{\circ}\right)\right)\right]\right) \ldots \ldots . .(43)
\end{aligned}
$$

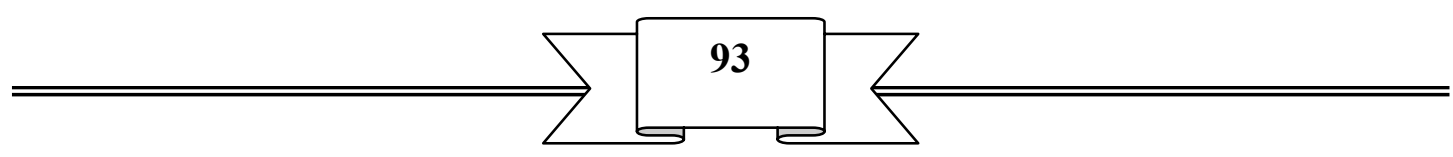




$$
\begin{aligned}
& \text { - R.N.Butris \& Merna Adel Aziz } \\
& u\left(t, x_{\circ}, y_{\circ}\right)=y_{\circ} e^{C t}+L^{2}\left(e ^ { C ( t - s ) } \left[C u\left(t, x_{\circ}, y_{\circ}\right)+D(t)\left(u\left(t, x_{\circ}, y_{\circ}\right)+\dot{u}\left(t, x_{\circ}, y_{\circ}\right)\right)+\right.\right. \\
& \left.\left.+g\left(t, r\left(t, x_{\circ}, y_{\circ}\right), u\left(t, x_{\circ}, y_{\circ}\right), \dot{r}\left(t, x_{\circ}, y_{\circ}\right), \dot{u}\left(t, x_{\circ}, y_{\circ}\right)\right)\right]\right) \\
& Q^{*}\left(t, x_{\circ}, y_{\circ}\right)=C u\left(t, x_{\circ}, y_{\circ}\right)+D(t)\left(u\left(t, x_{\circ}, y_{\circ}\right)+\dot{u}\left(t, x_{\circ}, y_{\circ}\right)\right)+ \\
& +g\left(t, r\left(t, x_{\circ}, y_{\circ}\right), u\left(t, x_{\circ}, y_{\circ}\right), \dot{r}\left(t, x_{\circ}, y_{\circ}\right), \dot{u}\left(t, x_{\circ}, y_{\circ}\right)\right) \\
& \dot{u}\left(t, x_{\circ}, y_{\circ}\right)=L\left(e ^ { C ( t - s ) } \left[C u\left(t, x_{\circ}, y_{\circ}\right)+D(t)\left(u\left(t, x_{\circ}, y_{\circ}\right)+\dot{u}\left(t, x_{\circ}, y_{\circ}\right)\right)+\right.\right. \\
& \left.\left.+g\left(t, r\left(t, x_{\circ}, y_{\circ}\right), u\left(t, x_{\circ}, y_{\circ}\right), \dot{r}\left(t, x_{\circ}, y_{\circ}\right), \dot{u}\left(t, x_{\circ}, y_{\circ}\right)\right)\right]\right)
\end{aligned}
$$

We must prove that $r\left(t, x_{\circ}, y_{\circ}\right)=x\left(t, x_{\circ}, y_{\circ}\right), \dot{r}\left(t, x_{\circ}, y_{\circ}\right)=\dot{x}\left(t, x_{\circ}, y_{\circ}\right)$, $y\left(t, x_{\circ}, y_{\circ}\right)=u\left(t, x_{\circ}, y_{\circ}\right), \dot{y}\left(t, x_{\circ}, y_{\circ}\right)=\dot{u}\left(t, x_{\circ}, y_{\circ}\right)$ and this from $\left\|x\left(t, x_{\circ}, y_{\circ}\right)-r\left(t, x_{\circ}, y_{\circ}\right)\right\| \leq \frac{T}{2}\left(\frac{T}{2} Q\left(N_{1}+H\right)+Q C_{2}\right)\left\|x\left(t, x_{\circ}, y_{\circ}\right)-r\left(t, x_{\circ}, y_{\circ}\right)\right\|+$
$+\frac{T}{2}\left(\frac{T}{2} Q H+Q C_{4}\right)\left\|\dot{x}_{1}\left(t, x_{\circ}, y_{\circ}\right)-\dot{r}\left(t, x_{\circ}, y_{\circ}\right)\right\|+\frac{T}{2} Q C_{3}\left\|y\left(t, x_{\circ}, y_{\circ}\right)-u\left(t, x_{\circ}, y_{\circ}\right)\right\|+$ $+\frac{T}{2} Q C_{5}\left\|\dot{y}\left(t, x_{\circ}, y_{\circ}\right)-\dot{u}\left(t, x_{\circ}, y_{\circ}\right)\right\|$

$\left\|\dot{x}\left(t, x_{\circ}, y_{\circ}\right)-\dot{r}\left(t, x_{\circ}, y_{\circ}\right)\right\| \leq\left(\frac{T}{2} Q\left(N_{1}+H\right)+Q C_{2}\right)\left\|x\left(t, x_{\circ}, y_{\circ}\right)-r\left(t, x_{\circ}, y_{\circ}\right)\right\|+$ $+\left(\frac{T}{2} Q H+Q C_{4}\right)\left\|\dot{x}_{1}\left(t, x_{\circ}, y_{\circ}\right)-\dot{r}\left(t, x_{\circ}, y_{\circ}\right)\right\|+Q C_{3}\left\|y\left(t, x_{\circ}, y_{\circ}\right)-u\left(t, x_{\circ}, y_{\circ}\right)\right\|+$

$$
+Q C_{5}\left\|\dot{y}\left(t, x_{\circ}, y_{\circ}\right)-\dot{u}\left(t, x_{\circ}, y_{\circ}\right)\right\|
$$

$$
\begin{aligned}
&\left\|y\left(t, x_{\circ}, y_{\circ}\right)-u\left(t, x_{\circ}, y_{\circ}\right)\right\| \leq \frac{T}{2} R \delta_{2}\left\|x\left(t, x_{\circ}, y_{\circ}\right)-r\left(t, x_{\circ}, y_{\circ}\right)\right\|+ \\
&+\frac{T}{2} R \delta_{4}\left\|\dot{x}\left(t, x_{\circ}, y_{\circ}\right)-\dot{r}\left(t, x_{\circ}, y_{\circ}\right)\right\|+\frac{T}{2}\left(\frac{T}{2} R\left(N_{2}+J\right)+R \delta_{3}\right)\left\|y\left(t, x_{\circ}, y_{\circ}\right)-u\left(t, x_{\circ}, y_{\circ}\right)\right\|+ \\
&+\frac{T}{2}\left(\frac{T}{2} R J+R \delta_{5}\right)\left\|\dot{y}\left(t, x_{\circ}, y_{\circ}\right)-\dot{u}\left(t, x_{\circ}, y_{\circ}\right)\right\| \quad \ldots \ldots . .(48)
\end{aligned}
$$

$$
\begin{aligned}
&\left\|\dot{y}\left(t, x_{\circ}, y_{\circ}\right)-\dot{u}\left(t, x_{\circ}, y_{\circ}\right)\right\| \leq R \delta_{2}\left\|x\left(t, x_{\circ}, y_{\circ}\right)-r\left(t, x_{\circ}, y_{\circ}\right)\right\|+ \\
&+R \delta_{4}\left\|\dot{x}\left(t, x_{\circ}, y_{\circ}\right)-\dot{r}\left(t, x_{\circ}, y_{\circ}\right)\right\|+\left(\frac{T}{2} R\left(N_{2}+J\right)+R \delta_{3}\right)\left\|y\left(t, x_{\circ}, y_{\circ}\right)-u\left(t, x_{\circ}, y_{\circ}\right)\right\|+ \\
&+\left(\frac{T}{2} R J+R \delta_{5}\right)\left\|\dot{y}\left(t, x_{\circ}, y_{\circ}\right)-\dot{u}\left(t, x_{\circ}, y_{\circ}\right)\right\|
\end{aligned}
$$

From (46),(47),(48),(49) we find

$\Longrightarrow \underset{ }{9} \stackrel{94}{ }$




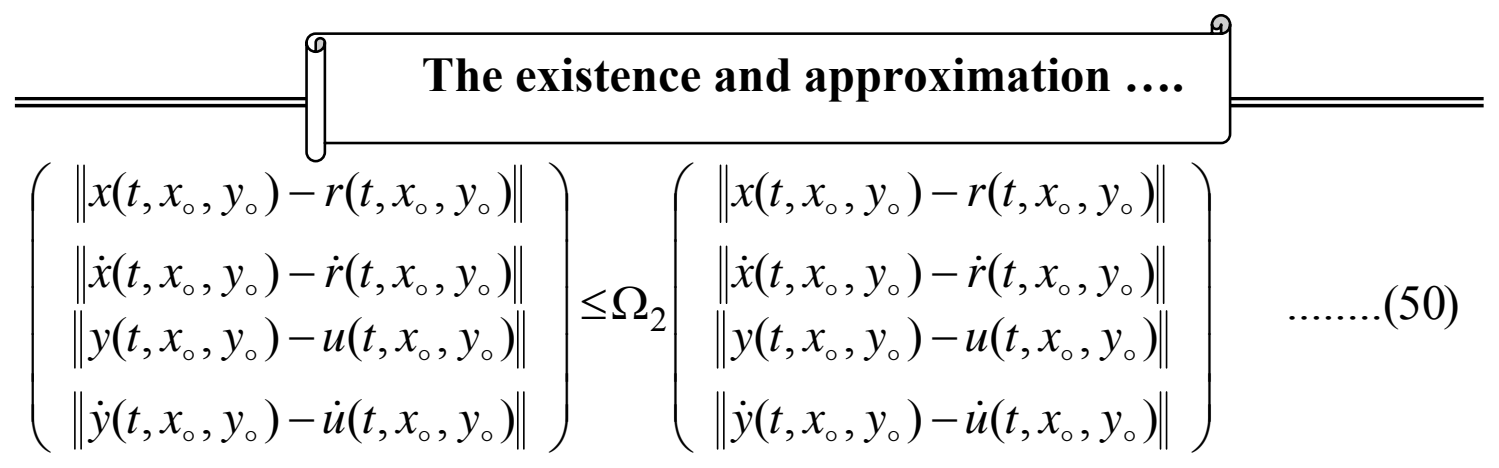

and by repetition (50) we obtain

$$
\left(\begin{array}{l}
\left\|x\left(t, x_{\circ}, y_{\circ}\right)-r\left(t, x_{\circ}, y_{\circ}\right)\right\| \\
\left\|\dot{x}\left(t, x_{\circ}, y_{\circ}\right)-\dot{r}\left(t, x_{\circ}, y_{\circ}\right)\right\| \\
\left\|y\left(t, x_{\circ}, y_{\circ}\right)-u\left(t, x_{\circ}, y_{\circ}\right)\right\| \\
\left\|\dot{y}\left(t, x_{\circ}, y_{\circ}\right)-\dot{u}\left(t, x_{\circ}, y_{\circ}\right)\right\|
\end{array}\right) \leq \Omega_{2}^{m}\left(\begin{array}{c}
\left\|x\left(t, x_{\circ}, y_{\circ}\right)-r\left(t, x_{\circ}, y_{\circ}\right)\right\| \\
\left\|\dot{x}\left(t, x_{\circ}, y_{\circ}\right)-\dot{r}\left(t, x_{\circ}, y_{\circ}\right)\right\| \\
\left\|y\left(t, x_{\circ}, y_{\circ}\right)-u\left(t, x_{\circ}, y_{\circ}\right)\right\| \\
\left\|\dot{y}\left(t, x_{\circ}, y_{\circ}\right)-\dot{u}\left(t, x_{\circ}, y_{\circ}\right)\right\|
\end{array}\right)
$$

but from the condition (12) we get $\Omega_{2}^{m} \rightarrow 0$ when $m \rightarrow \infty$ and then we have from the last inequality that

$$
\begin{aligned}
& x\left(t, x_{\circ}, y_{\circ}\right)=r\left(t, x_{\circ}, y_{\circ}\right), y\left(t, x_{\circ}, y_{\circ}\right)=u\left(t, x_{\circ}, y_{\circ}\right) \\
& \dot{x}\left(t, x_{\circ}, y_{\circ}\right)=\dot{r}\left(t, x_{\circ}, y_{\circ}\right), \dot{y}\left(t, x_{\circ}, y_{\circ}\right)=\dot{u}\left(t, x_{\circ}, y_{\circ}\right)
\end{aligned}
$$

that is each of $x\left(t, x_{\circ}, y_{\circ}\right), y\left(t, x_{\circ}, y_{\circ}\right)$ are unique solution for the system

\section{Section Two : The existence of the periodic solution for the system (1)}

The problem of the existence of the periodic solution for the system (1) is connected with unique form with existence zero for all of the two functions

$\Delta_{1}^{*}=\Delta_{1}^{*}\left(x_{\circ}, y_{\circ}\right), \Delta_{2}^{*}=\Delta_{2}^{*}\left(x_{\circ}, y_{\circ}\right)$ in the form below :

$\Delta_{1}^{*}: D_{f} \times D_{g} \rightarrow R^{n}$,

$\Delta_{1}^{*}\left(x_{\circ}, y_{\circ}\right)=\frac{1}{T} \int_{0}^{T} L\left(e^{A(t-s)} H_{\infty}\left(t, x_{\circ}, y_{\circ}\right)\right) d t \quad$,

$\Delta_{2}^{*}: D_{f} \times D_{g} \rightarrow R^{n}$,

$\Delta_{2}^{*}\left(x_{\circ}, y_{\circ}\right)=\frac{1}{T} \int_{0}^{T} L\left(e^{C(t-s)} Q_{\infty}\left(t, x_{\circ}, y_{\circ}\right)\right) d t \quad$,

where $x_{\infty}\left(t, x_{\circ}, y_{\circ}\right)$ it is the end of the sequence $x_{m}\left(t, x_{\circ}, y_{\circ}\right)$ and $y_{\infty}\left(t, x_{\circ}, y_{\circ}\right)$

it is the end of the sequence $y_{m}\left(t, x_{\circ}, y_{\circ}\right)$. The existence of these two functions are impossible to prove except by successive approximation from the following two sequences of the functions

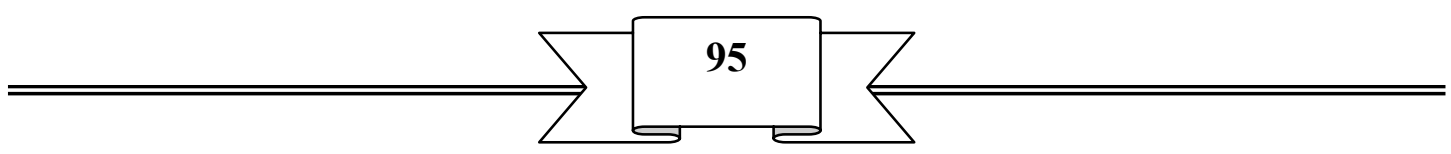




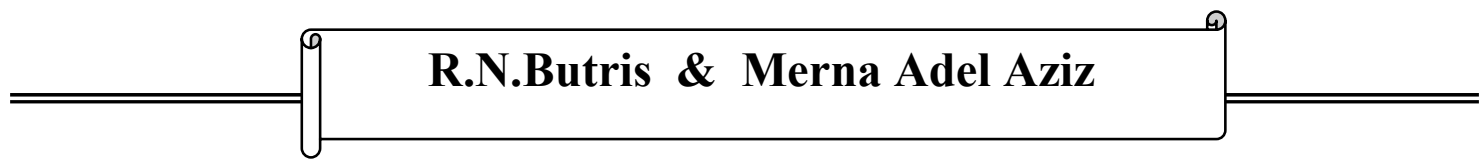

$\Delta_{1 m}^{*}: D_{f} \times D_{g} \rightarrow R^{n}$,

$\Delta_{1 m}^{*}\left(x_{\circ}, y_{\circ}\right)=\frac{1}{T} \int_{0}^{T} L\left(e^{A(t-s)} H_{m}\left(t, x_{\circ}, y_{\circ}\right)\right) d t \quad$,

$\Delta_{2 m}^{*}: D_{f} \times D_{f} \rightarrow R^{n}$,

$\Delta_{2 m}^{*}\left(x_{\circ}, y_{\circ}\right)=\frac{1}{T} \int_{0}^{T} L\left(e^{C(t-s)} Q_{m}\left(t, x_{\circ}, y_{\circ}\right)\right) d t \quad$,

where $\mathrm{m}=0,1,2, \ldots \ldots$.

\section{Theorem 2:-}

If were the assumptions and the conditions of the theorem 1 be given then the following inequalities :

$$
\begin{aligned}
& \left\|\Delta_{1}^{*}\left(x_{\circ}, y_{\circ}\right)-\Delta_{1 m}^{*}\left(x_{\circ}, y_{\circ}\right)\right\| \leq
\end{aligned}
$$

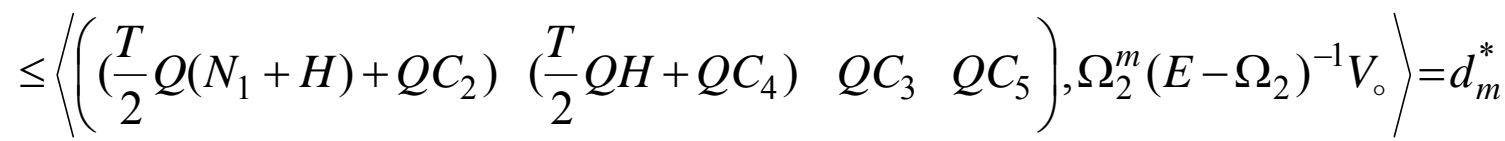

$$
\begin{aligned}
& \left\|\Delta_{2}^{*}\left(x_{\circ}, y_{\circ}\right)-\Delta_{2 m}^{*}\left(x_{\circ}, y_{\circ}\right)\right\| \leq \\
& \leq\left\langle\left(\begin{array}{llll}
R \delta_{2} & R \delta_{4} & \left(\frac{T}{2} R\left(N_{2}+J\right)+R \delta_{3}\right) & \left(\frac{T}{2} R J+R \delta_{5}\right)
\end{array}\right), \Omega_{2}^{m}\left(E-\Omega_{2}\right)^{-1} V_{\circ}\right\rangle=b_{m}^{*}
\end{aligned}
$$

satisfies for $m \geq 0, x_{\circ} \in D_{f}, y_{\circ} \in D_{g}$ and $d_{m}^{*}, b_{m}^{*}$ are positive numerical constants.

and $\langle$.$\rangle denote to the non cross product in the Euclidean space R^{n}$.

\section{Proof :-}

By the equations (51), (53) and the conditions (30) we have

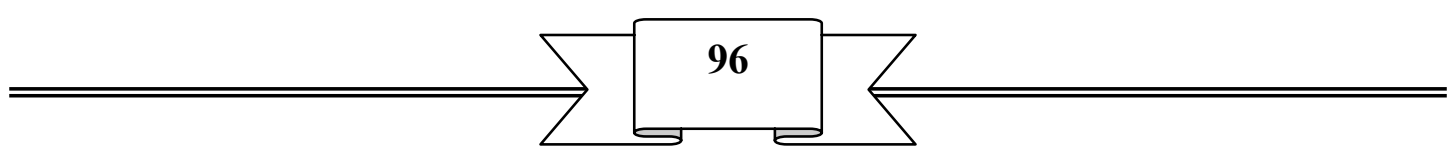




$$
\begin{aligned}
& \hline \hline \underbrace{q} \text { The existence and approximation .... } \\
& \left\|\Delta_{1}^{*}\left(x_{\circ}, y_{\circ}\right)-\Delta_{1 m}^{*}\left(x_{\circ}, y_{\circ}\right)\right\| \leq \\
& \leq \beta_{1}(t)\left\|e^{A(t-s)}\right\|\left[\|A\|\left\|x_{\infty}\left(t, x_{\circ}, y_{\circ}\right)-x_{m}\left(t, x_{\circ}, y_{\circ}\right)\right\|+\right. \\
& \left.+\|B(t)\|\left(\left\|x_{\infty}\left(t, x_{\circ}, y_{\circ}\right)-x_{m}\left(t, x_{\circ}, y_{\circ}\right)\right\|+\left\|\dot{x}_{\infty}\left(t, x_{\circ}, y_{\circ}\right)-\dot{x}_{m}\left(t, x_{\circ}, y_{\circ}\right)\right\|\right)\right]+ \\
& +\left\|e^{A(t-s)}\right\| \int_{0}^{t}\left[K_{1}(s)\left\|x_{\infty}\left(s, x_{\circ}, y_{\circ}\right)-x_{m}\left(s, x_{\circ}, y_{\circ}\right)\right\|+K_{2}(s)\left\|y_{\infty}\left(s, x_{\circ}, y_{\circ}\right)-y_{m}\left(s, x_{\circ}, y_{\circ}\right)\right\|+\right. \\
& \left.\quad+K_{3}(s)\left\|\dot{x}_{\infty}\left(s, x_{\circ}, y_{\circ}\right)-\dot{x}_{m}\left(t, x_{\circ}, y_{\circ}\right)\right\|+K_{4}(s)\left\|\dot{y}_{\infty}\left(s, x_{\circ}, y_{\circ}\right)-\dot{y}_{m}\left(s, x_{\circ}, y_{\circ}\right)\right\|\right] d s \\
& \leq\left\langle\left(\left(\frac{T}{2} Q\left(N_{1}+H\right)+Q C_{2}\right) \quad\left(\frac{T}{2} Q H+Q C_{4}\right) \quad Q C_{3} \quad Q C_{5}\right), \Omega_{2}^{m}\left(E-\Omega_{2}\right)^{-1} V_{\circ}\right\rangle=d_{m}^{*}
\end{aligned}
$$

also by the equations (52), (54) and the conditions (30) we have

$$
\begin{aligned}
& \left\|\Delta_{2}^{*}\left(x_{\circ}, y_{\circ}\right)-\Delta_{2 m}^{*}\left(x_{\circ}, y_{\circ}\right)\right\| \leq \\
& \leq \beta_{2}(t)\left\|e^{C(t-s)}\right\|\left[\|C\|\left\|y_{\infty}\left(t, x_{\circ}, y_{\circ}\right)-y_{m}\left(t, x_{\circ}, y_{\circ}\right)\right\|+\right. \\
& \left.+\|D(s)\|\left(\left\|y_{\infty}\left(t, x_{\circ}, y_{\circ}\right)-y_{m}\left(t, x_{\circ}, y_{\circ}\right)\right\|+\left\|\dot{y}_{\infty}\left(t, x_{\circ}, y_{\circ}\right)-\dot{y}_{m}\left(t, x_{\circ}, y_{\circ}\right)\right\|\right)\right]+ \\
& +\left\|e^{C(t-s)}\right\| \int_{0}^{t}\left[L_{1}(s)\left\|x_{\infty}\left(s, x_{\circ}, y_{\circ}\right)-x_{m}\left(t, x_{\circ}, y_{\circ}\right)\right\|+L_{2}(s)\left\|y_{\infty}\left(s, x_{\circ}, y_{\circ}\right)-y_{m}\left(t, x_{\circ}, y_{\circ}\right)\right\|+\right. \\
& \left.+L_{3}(s)\left\|\dot{x}_{\infty}\left(s, x_{\circ}, y_{\circ}\right)-\dot{x}_{m}\left(t, x_{\circ}, y_{\circ}\right)\right\|+L_{4}(s)\left\|\dot{y}_{\infty}\left(s, x_{\circ}, y_{\circ}\right)-\dot{y}_{m}\left(t, x_{\circ}, y_{\circ}\right)\right\|\right] d s
\end{aligned}
$$

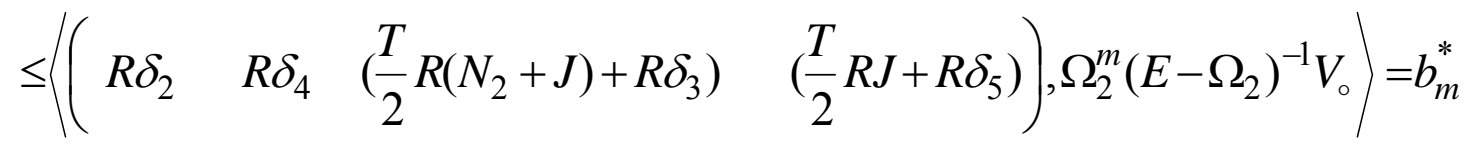

By the helping of the theorem 2 we introduce the following theorem and take into consideration the truth of the two inequalities (55) and (56)for $m \geq 0$.

\section{Theorem 3:-}

Let the right hand side from the system (1) are defined in the domain

$$
a \leq x, \dot{x} \leq b, c \leq y, \dot{y} \leq d, 0 \leq t \leq T \text { on } \mathrm{R}^{1} \text { and assuming that the two }
$$

sequences of the functions (53),(54) satisfies the following inequalities :

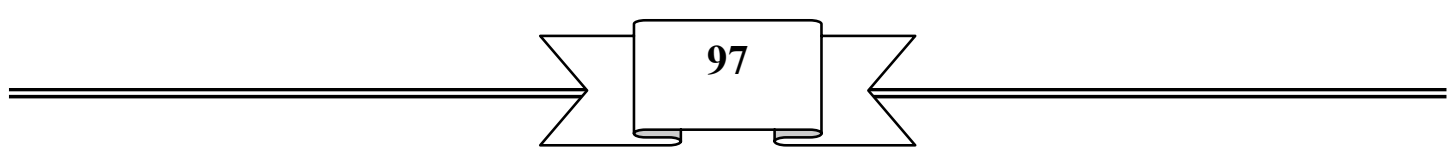




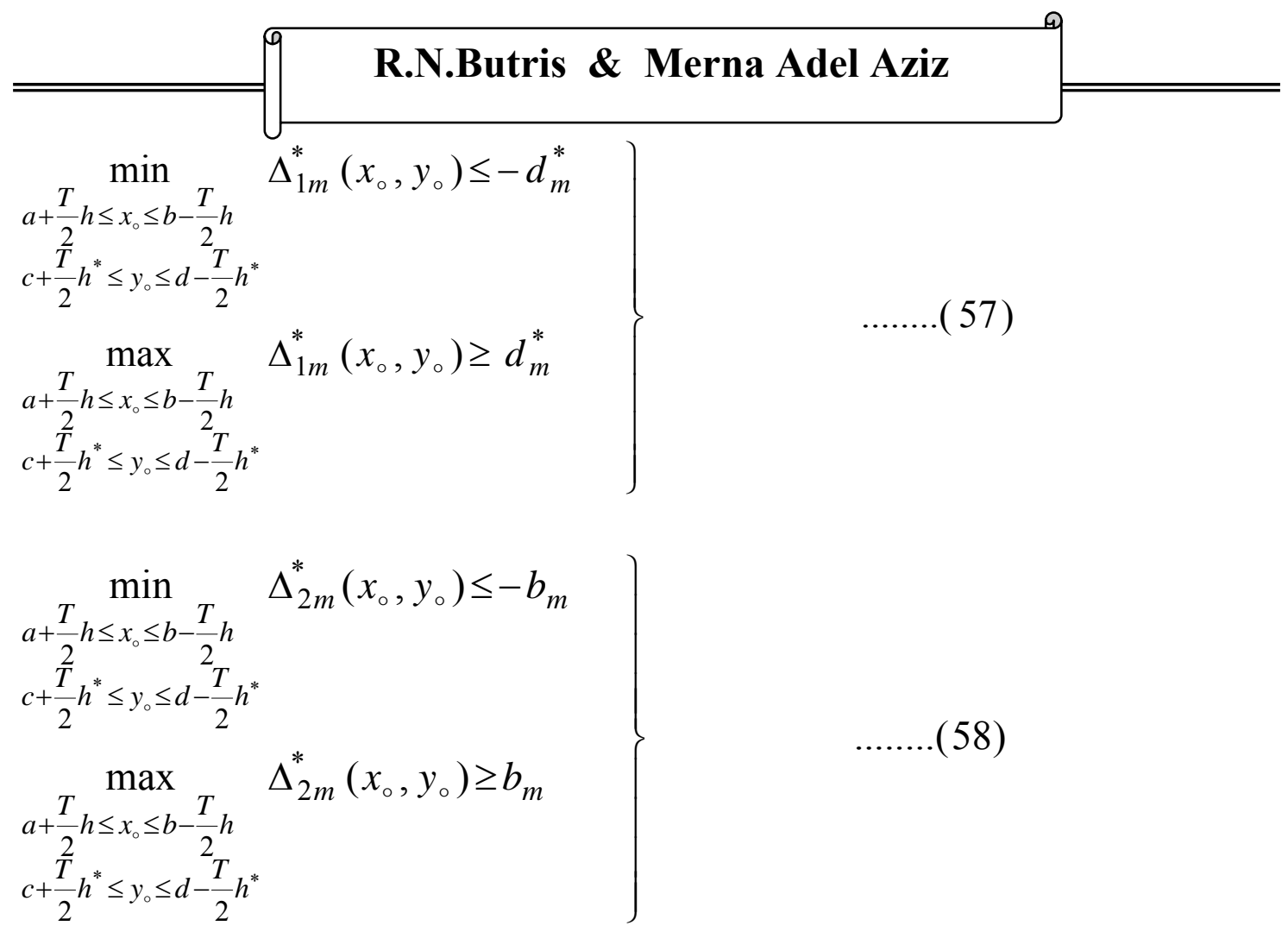

For all

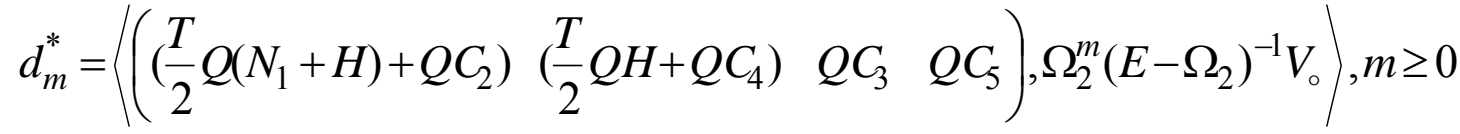

$$
\begin{aligned}
& \left.b_{m}^{*}=\left\langle\begin{array}{llll}
R \delta_{2} & R \delta_{4} & \left(\frac{T}{2} R\left(N_{2}+J\right)+R \delta_{3}\right) & \left(\frac{T}{2} R J+R \delta_{5}\right)
\end{array}\right), \Omega_{2}^{m}\left(E-\Omega_{2}\right)^{-1} V_{\circ}\right\rangle, m \geq 0 \\
& N^{*}=R^{2} J \sigma_{\circ}, N=Q^{2} H \delta_{\circ}, h^{*}=\frac{T}{2} N^{*}+R \delta_{1}, h=\frac{T}{2} N+Q C_{1}
\end{aligned}
$$

then the system (1) have periodic solutions

$$
\begin{aligned}
& x=x\left(t, x_{\circ}, y_{\circ}\right), y=y\left(t, x_{\circ}, y_{\circ}\right) \\
& \text { for } x_{\circ} \in\left[a+\frac{T}{2} h, b-\frac{T}{2} h\right], y_{\circ} \in\left[c+\frac{T}{2} h^{*}, d-\frac{T}{2} h^{*}\right] .
\end{aligned}
$$

\section{Proof :-}

Let $x_{1}, x_{2}$ be any two points in the interval $\left[a+\frac{T}{2} h, b-\frac{T}{2} h\right], y_{2}, y_{1}$ be any two points in the interval $\left[c+\frac{T}{2} h^{*}, d-\frac{T}{2} h^{*}\right]$ so that

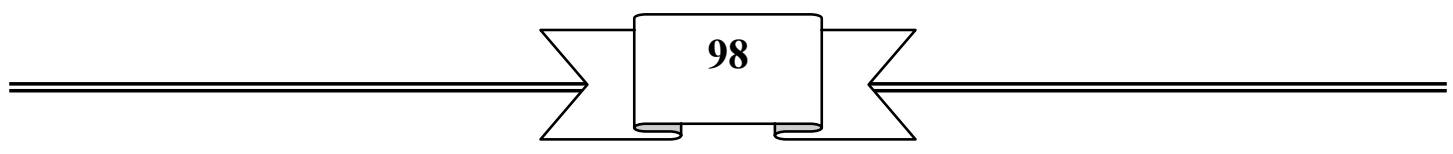




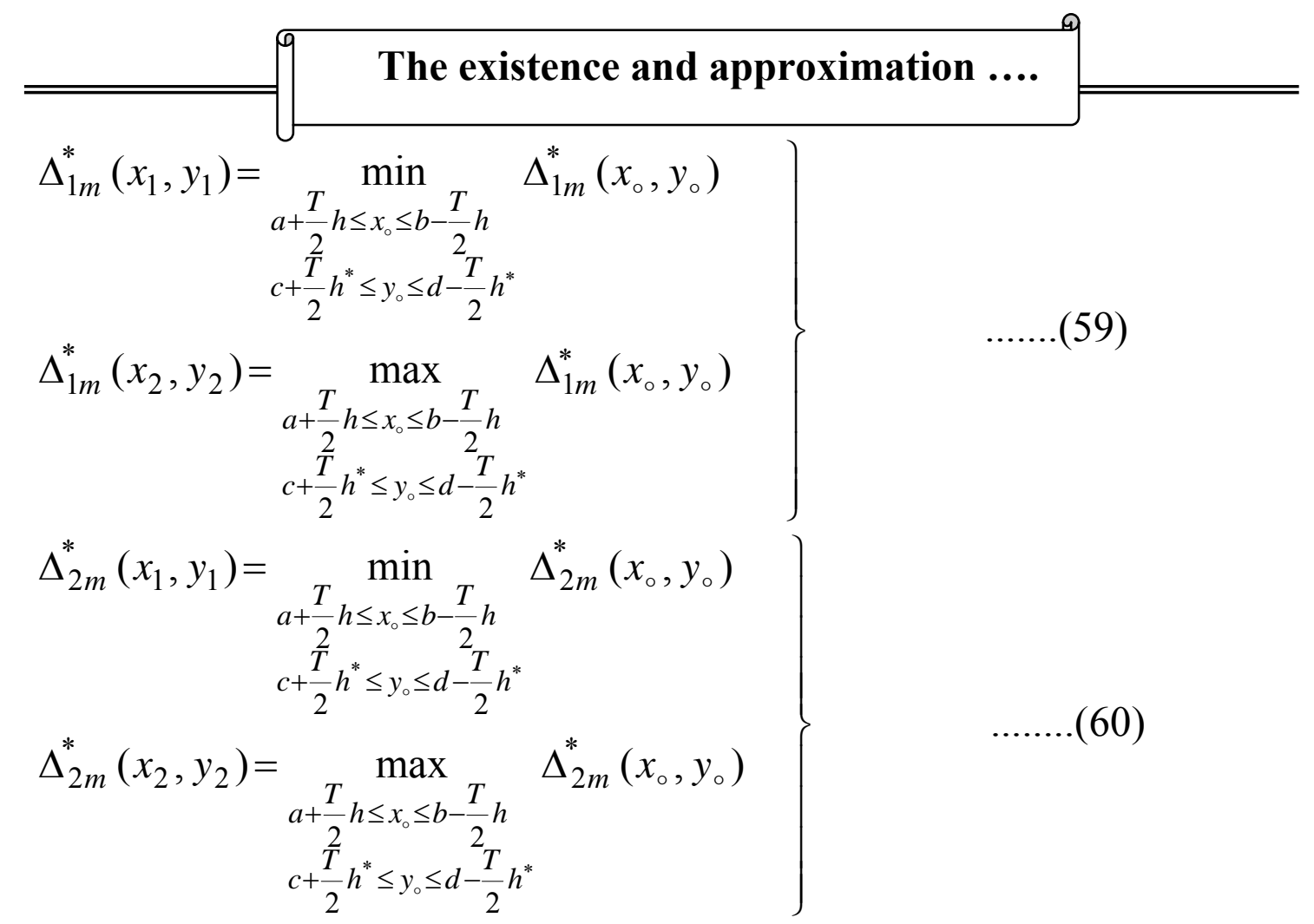

by using the inequalities (55),(56),(57),(58) we have

$$
\left.\left.\begin{array}{l}
\Delta_{1}^{*}\left(x_{1}, y_{1}\right)=\Delta_{1 m}^{*}\left(x_{1}, y_{1}\right)+\left(\Delta_{1}^{*}\left(x_{1}, y_{1}\right)-\Delta_{1 m}^{*}\left(x_{1}, y_{1}\right)\right)<0 \\
\Delta_{1}^{*}\left(x_{2}, y_{2}\right)=\Delta_{1 m}^{*}\left(x_{2}, y_{2}\right)+\left(\Delta_{1}^{*}\left(x_{2}, y_{2}\right)-\Delta_{1 m}^{*}\left(x_{2}, y_{2}\right)\right)>0
\end{array}\right\} \begin{array}{l}
\Delta_{2}^{*}\left(x_{1}, y_{1}\right)=\Delta_{2 m}^{*}\left(x_{1}, y_{1}\right)+\left(\Delta_{2}^{*}\left(x_{1}, y_{1}\right)-\Delta_{2 m}^{*}\left(x_{1}, y_{1}\right)\right)<0 \\
\Delta_{2}^{*}\left(x_{2}, y_{2}\right)=\Delta_{2 m}^{*}\left(x_{2}, y_{2}\right)+\left(\Delta_{2}^{*}\left(x_{2}, y_{2}\right)-\Delta_{2 m}^{*}\left(x_{2}, y_{2}\right)\right)>0
\end{array}\right\}
$$

and from the continuity of the two functions $\Delta_{1}^{*}\left(x_{\circ}, y_{\circ}\right), \Delta_{2}^{*}\left(x_{\circ}, y_{\circ}\right)$ and the two inequalities $(61),(62)$ then there exist an isolated single point $\left(x_{\infty}, y_{\infty}\right)=\left(x_{\circ}, y_{\circ}\right)$ and $x_{\infty} \in\left[x_{1}, x_{2}\right], y_{\infty} \in\left[y_{1}, y_{2}\right]$ where $\Delta_{1}^{*}\left(x_{\circ}, y_{\circ}\right)=0, \Delta_{2}^{*}\left(x_{\circ}, y_{\circ}\right)=0$

this mean that for the system (1) periodic solution $x=\left(t, x_{\circ}, y_{\circ}\right), y=\left(t, x_{\circ}, y_{\circ}\right)$ for $x_{\circ} \in\left[a+\frac{T}{2} h, b-\frac{T}{2} h\right], y_{\circ} \in\left[c+\frac{T}{2} h^{*}, d-\frac{T}{2} h^{*}\right]$.

\section{Remark 1 :-}

We completed the formulation for the text of the theorem 3 with the proof when $R^{n}=R^{1}$ that is when $x_{\circ}, y_{\circ}$ are two non vectors quantities

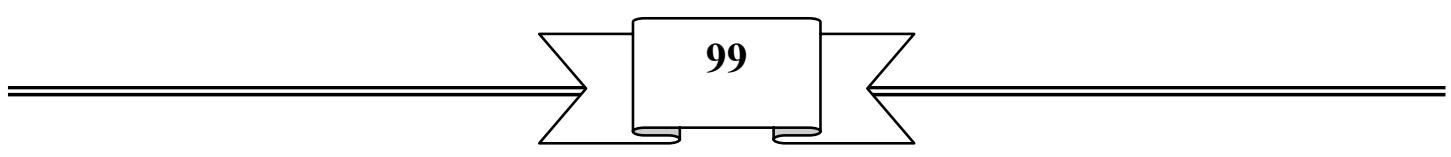




\section{R.N.Butris \& Merna Adel Aziz}

\section{Theorem 4 :-}

Let

$\Delta_{1}^{*}: D_{f} \times D_{g} \rightarrow R^{n}$

$\Delta_{1}^{*}\left(x_{\circ}, y_{\circ}\right)=\frac{1}{T} \int_{0}^{T} L\left(e^{A(t-s)} H_{\infty}\left(t, x_{\circ}, y_{\circ}\right)\right) d t$

$\Delta_{2}^{*}: D_{f} \times D_{g} \rightarrow R^{n}$

$\Delta_{2}^{*}\left(x_{\circ}, y_{\circ}\right)=\frac{1}{T} \int_{0}^{T} L\left(e^{C(t-s)} Q_{\infty}\left(t, x_{\circ}, y_{\circ}\right)\right) d t$

where $x_{\infty}\left(t, x_{\circ}, y_{\circ}\right)$ it is the end of the periodic sequence (25) and the function

$y_{\infty}\left(t, x_{\circ}, y_{\circ}\right)$ it is the end of the periodic sequence (26) then the following inequalities

$\left\|\Delta_{1}^{*}\left(x_{\circ}, y_{\circ}\right)\right\| \leq M_{13}$

$$
M_{13}=\frac{T}{2} Q\left(N_{1}+H\right) M_{9}\left[\delta_{\circ} Q+\frac{T^{2}}{4} Q^{2} H C_{1} M_{3}+\frac{T}{2} Q C_{1}+\frac{T}{2} Q H M_{3} \delta_{\circ} Q+\right.
$$

where $\left.\quad+\frac{T^{3}}{8} Q^{3} H^{2} M_{3}^{2} C_{1}+\frac{T^{2}}{4} Q^{2} H C_{1} M_{3}\right]+Q C_{1}\left(\frac{T}{2} Q H M_{3}+1\right)$

$$
\begin{gathered}
M_{3}=\left(1-\frac{T}{2} Q H\right)^{-1}, M_{9}=\left(1-\frac{T^{2}}{4} Q\left(N_{1}+H\right)\left(1+\frac{T}{2} Q H M_{3}\right)\right)^{-1} \\
\left\|\Delta_{2}^{*}\left(x_{\circ}, y_{\circ}\right)\right\| \leq M_{14}
\end{gathered}
$$

where

$$
\begin{aligned}
M_{14} & =\frac{T}{2} R\left(N_{2}+J\right) M_{11}\left[\sigma_{\circ} R+\frac{T^{2}}{4} R^{2} J \delta_{1} M_{5}+\frac{T}{2} R \delta_{1}+\frac{T}{2} R J M_{5} \sigma_{\circ} R+\right. \\
& \left.+\frac{T^{3}}{8} R^{3} J^{2} M_{5}^{2} \delta_{1}+\frac{T^{2}}{4} R^{2} J M_{5} \delta_{1}\right]+R \delta_{1}\left(\frac{T}{2} R J M_{5}+1\right) \\
M_{5}= & \left(1-\frac{T}{2} R J\right)^{-1}, M_{11}=\left(1-\frac{T^{2}}{4} R\left(N_{2}+J\right)\left(1+\frac{T}{2} R J M_{5}\right)\right)^{-1}
\end{aligned}
$$

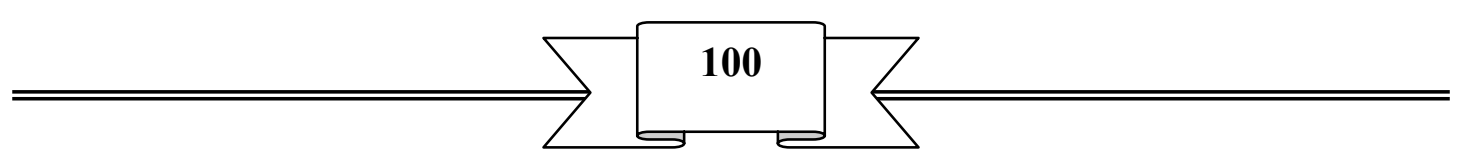




\section{The existence and approximation ....}

$$
\begin{aligned}
\left\|\Delta_{1}^{*}\left(x_{\circ}^{1}, y_{\circ}^{1}\right)-\Delta_{1}^{*}\left(x_{\circ}^{2}, y_{\circ}^{2}\right)\right\| \leq & {\left[\frac{T}{2} E_{5} W_{1} W_{3} W_{5} W_{6} W_{9} W_{10} W_{11} \gamma_{1}+W_{1} W_{5} W_{8} \gamma_{2}\right]\left\|x_{\circ}^{1}-x_{\circ}^{2}\right\| Q+} \\
& +\left[W_{3} W_{6} W_{9} W_{10} W_{11} \gamma_{1}\right]\left\|y_{\circ}^{1}-y_{\circ}^{2}\right\| R
\end{aligned}
$$

$\left\|\Delta_{2}^{*}\left(x_{\circ}^{1}, y_{\circ}^{1}\right)-\Delta_{2}^{*}\left(x_{\circ}^{2}, y_{\circ}^{2}\right)\right\| \leq\left[\frac{T}{2} E_{5} W_{1} W_{3} W_{5} W_{6} W_{9} W_{10} W_{11} \gamma_{3}+W_{1} W_{5} W_{8} \gamma_{4}\right]\left\|x_{\circ}^{1}-x_{\circ}^{2}\right\| Q+$

$$
+\left[W_{3} W_{6} W_{9} W_{10} W_{11} \gamma_{3}\right]\left\|y_{\circ}^{1}-y_{\circ}^{2}\right\| R
$$

satisfies for $x_{\circ}, x_{\circ}^{1}, x_{\circ}^{2} \in D_{f}, y_{\circ}, y_{\circ}^{1}, y_{\circ}^{2} \in D_{g}$

Where

$$
\begin{aligned}
& E_{1}=\frac{T}{2} Q\left(N_{1}+H\right)+Q C_{2}, E_{2}=\frac{T}{2} Q H+Q C_{4}, E_{3}=Q C_{3}, E_{4}=Q C_{5} \\
& E_{5}=R \delta_{2}, E_{6}=R \delta_{4}, E_{7}=\frac{T}{2} R\left(N_{2}+J\right)+R \delta_{3}, E_{8}=\frac{T}{2} R J+R \delta_{5} \\
& \gamma_{1}=E_{1} W_{5} W_{8} a_{1}+E_{1} E_{2} W_{2} W_{5} W_{8} a_{1}+E_{2} E_{3} W_{2}+E_{2} E_{4} W_{2} W_{5} W_{7} W_{8} a_{1} a_{4}+ \\
& +E_{2} E_{4} W_{2} W_{7} a_{5}+E_{3}+E_{4} W_{5} W_{7} W_{8} a_{1} a_{4}+E_{4} W_{7} a_{5} \\
& \gamma_{2}=E_{1} W_{5} W_{8} W_{10} W_{11} a_{1} a_{7}+E_{1}+E_{1} E_{2} W_{2} W_{5} W_{8} W_{10} W_{11} a_{1} a_{7}+E_{1} E_{2} W_{2}+ \\
& +E_{2} E_{3} W_{2} W_{10} W_{11} a_{7}+E_{2} E_{4} W_{2} W_{5} W_{7} W_{8} W_{10} W_{11} a_{1} a_{4} a_{7}+E_{2} E_{4} W_{2} W_{7} a_{4}+ \\
& +E_{2} E_{4} W_{2} W_{7} W_{10} W_{11} a_{5} a_{7}+E_{3} W_{10} W_{11} a_{7}+E_{4} W_{5} W_{7} W_{8} W_{10} W_{11} a_{1} a_{4} a_{7}+ \\
& +E_{4} W_{7} a_{4}+E_{4} W_{7} W_{10} W_{11} a_{5} a_{7} \\
& \gamma_{3}=E_{5} W_{5} W_{8} a_{1}+E_{1} E_{6} W_{2} W_{5} W_{8} a_{1}+E_{6} E_{3} W_{2}+E_{4} E_{6} W_{2} W_{5} W_{7} W_{8} a_{1} a_{4}+ \\
& +E_{4} E_{6} W_{2} W_{7} a_{5}+E_{7}+E_{8} W_{5} W_{7} W_{8} a_{1} a_{4}+E_{8} W_{7} a_{5} \\
& \gamma_{4}=E_{5} W_{5} W_{8} W_{10} W_{11} a_{1} a_{7}+E_{5}+E_{1} E_{6} W_{2} W_{5} W_{8} W_{10} W_{11} a_{1} a_{7}+E_{1} E_{6} W_{2}+ \\
& +E_{3} E_{6} W_{2} W_{10} W_{11} a_{7}+E_{4} E_{6} W_{2} W_{5} W_{7} W_{8} W_{10} W_{11} a_{1} a_{4} a_{7}+E_{4} E_{6} W_{2} W_{7} a_{4}+ \\
& +E_{4} E_{6} W_{2} W_{7} W_{10} W_{11} a_{5} a_{7}+E_{7} W_{10} W_{11} a_{7}+E_{8} W_{5} W_{7} W_{8} W_{10} W_{11} a_{1} a_{4} a_{7}+ \\
& +E_{8} W_{7} a_{4}+E_{8} W_{7} W_{10} W_{11} a_{5} a_{7} \\
& W_{1}=\left(1-\frac{T}{2} E_{1}\right)^{-1}, W_{2}=\left(1-E_{2}\right)^{-1}, W_{3}=\left(1-\frac{T}{2} E_{7}\right)^{-1}, W_{4}=\left(1-E_{8}\right)^{-1}
\end{aligned}
$$

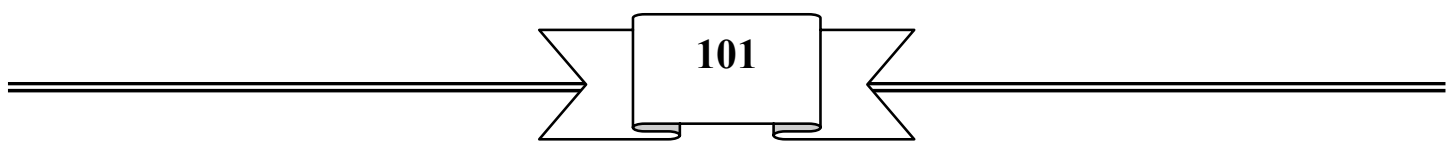




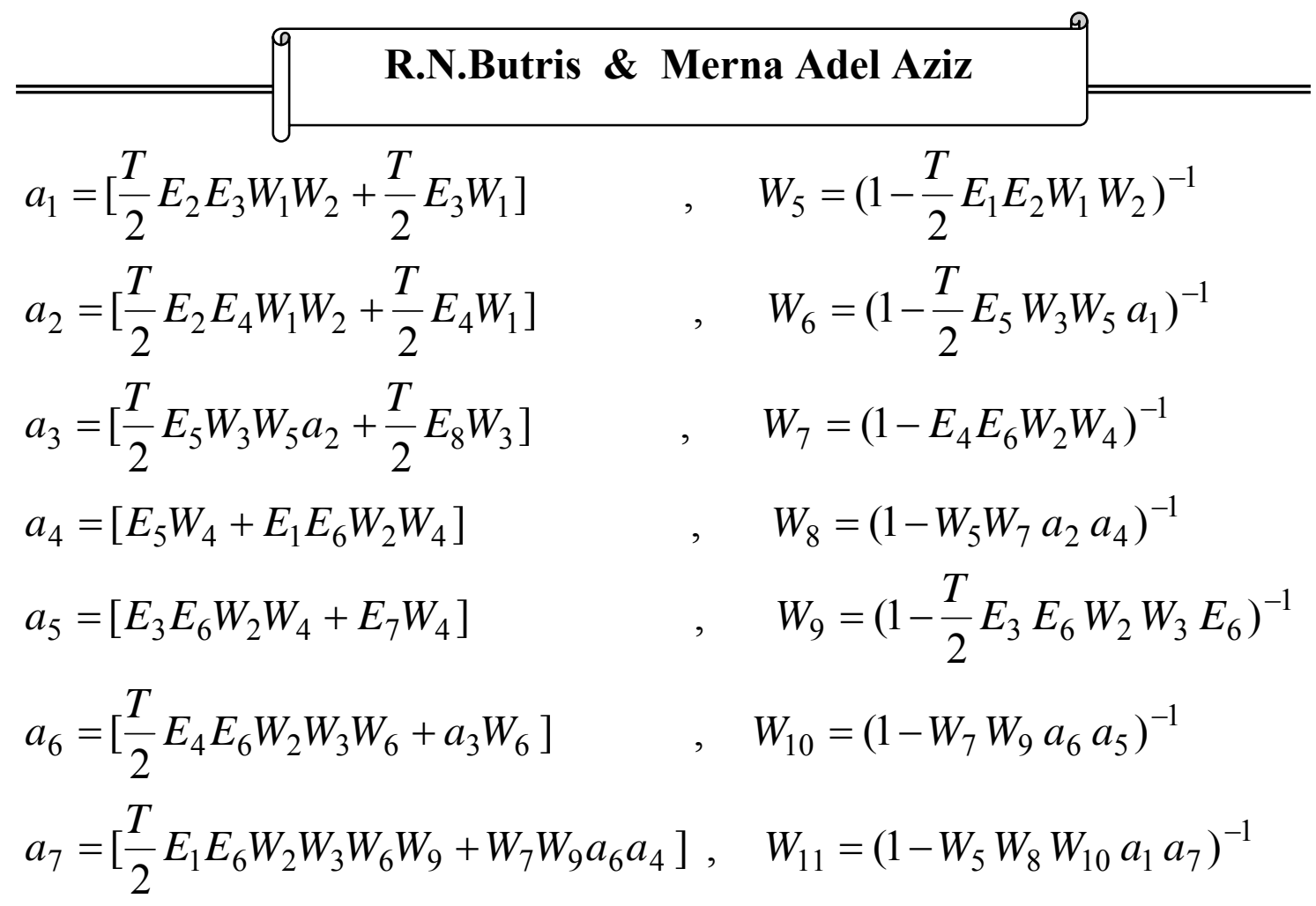

\section{Proof :-}

From properties of the function $x_{\infty}\left(t, x_{\circ}, y_{\circ}\right)$ and the function $y_{\infty}\left(t, x_{\circ}, y_{\circ}\right)$ that fixative by theorem 1 then each of the two functions $\Delta_{1}^{*}\left(x_{\circ}, y_{\circ}\right), \Delta_{2}^{*}\left(x_{\circ}, y_{\circ}\right)$, $x_{\circ} \in D_{f}, y_{\circ} \in D_{g}$ continuous and bounded by non negative constants $M_{13}, M_{14}$ on arrangement in the domain (2).

from the relation (63) we find that

$$
\left\|\Delta_{1}^{*}\left(x_{\circ}, y_{\circ}\right)\right\| \leq\left\|L\left(e^{A(t-s)} H_{\infty}\left(t,, x_{\circ}, y_{\circ}\right)\right)\right\|
$$

and by using lemma 1 we get

$$
\left\|\Delta_{1}^{*}\left(x_{\circ}, y_{\circ}\right)\right\| \leq \frac{T}{2} Q\left(N_{1}+H\right)\left\|x_{\infty}\left(t, x_{\circ}, y_{\circ}\right)\right\|+\frac{T}{2} Q H\left\|\dot{x}_{\infty}\left(t, x_{\circ}, y_{\circ}\right)\right\|+Q C_{1}
$$

since that $x_{\infty}\left(t, x_{\circ}, y_{\circ}\right)$ satisfy the integral equation (28) we find that

$$
\left\|x_{\infty}\left(t, x_{\circ}, y_{\circ}\right)\right\| \leq \delta_{\circ} Q+\frac{T^{2}}{4} Q\left(N_{1}+H\right)\left\|x_{\infty}\left(t, x_{\circ}, y_{\circ}\right)\right\|+\frac{T^{2}}{4} Q H\left\|\dot{x}_{\infty}\left(t, x_{\circ}, y_{\circ}\right)\right\|+\frac{T}{2} Q C_{1}
$$

also we have

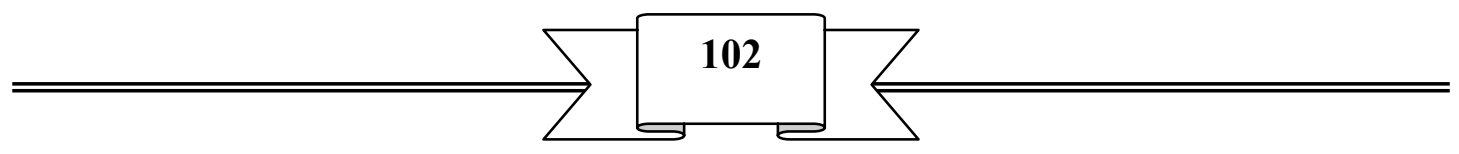




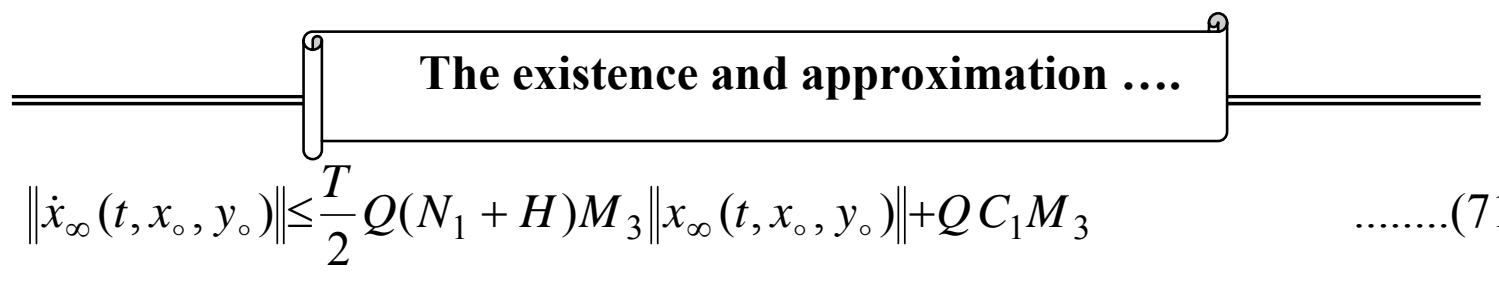

and by substitutions the inequality (71) in the inequality (70) we obtain $\left\|x_{\infty}\left(t, x_{\circ}, y_{\circ}\right)\right\| \leq \delta_{\circ} Q M_{9}+\frac{T}{2} Q C_{1}\left(\frac{T}{2} Q H M_{3}+1\right) M_{9}$

and by substitutions the inequality (72) in the inequality (71) we obtain

$$
\begin{aligned}
\left\|\dot{x}_{\infty}\left(t, x_{\circ}, y_{\circ}\right)\right\| \leq & \frac{T}{2} Q\left(N_{1}+H\right) M_{3} M_{9} \delta_{\circ} Q+\frac{T^{2}}{4} Q^{2}\left(N_{1}+H\right) C_{1} M_{3} M_{9}\left(\frac{T}{2} Q H M_{3}+1\right)+ \\
& +Q C_{1} M_{3}
\end{aligned}
$$

by substitutions the two inequalities (72),(73) in the inequality (70) we obtain (65).

From the relation (64) we find that

$$
\left\|\Delta_{2}^{*}\left(x_{\circ}, y_{\circ}\right)\right\| \leq\left\|L\left(e^{C(t-s)} Q_{\infty}\left(t, x_{\circ}, y_{\circ}\right)\right)\right\|
$$

and by using lemma 1 we get

$$
\left\|\Delta_{2}^{*}\left(x_{\circ}, y_{\circ}\right)\right\| \leq \frac{T}{2} R\left(N_{2}+J\right)\left\|y_{\infty}\left(t, x_{\circ}, y_{\circ}\right)\right\|+\frac{T}{2} R J\left\|\dot{y}_{\infty}\left(t, x_{\circ}, y_{\circ}\right)\right\|+R \delta_{1}
$$

since that $y_{\infty}\left(t, x_{\circ}, y_{\circ}\right)$ satisfy the integral equation (29) we find

$\left\|y_{\infty}\left(t, x_{\circ}, y_{\circ}\right)\right\| \leq \sigma_{\circ} R+\frac{T^{2}}{4} R\left(N_{2}+J\right)\left\|y_{\infty}\left(t, x_{\circ}, y_{\circ}\right)\right\|+\frac{T^{2}}{4} R J\left\|\dot{y}_{\infty}\left(t, x_{\circ}, y_{\circ}\right)\right\|+\frac{T}{2} R \delta_{1}$

also we have

$\left\|\dot{y}_{\infty}\left(t, x_{\circ}, y_{\circ}\right)\right\| \leq \frac{T}{2} R\left(N_{2}+J\right) M_{5}\left\|y_{\infty}\left(t, x_{\circ}, y_{\circ}\right)\right\|+R \delta_{1} M_{5}$

and by substitutions the inequality (76) in the inequality (75) we obtain $\left\|y_{\infty}\left(t, x_{\circ}, y_{\circ}\right)\right\| \leq \sigma_{\circ} R M_{11}+\frac{T}{2} R \delta_{1}\left(\frac{T}{2} R J M_{5}+1\right) M_{11}$

and by substitutions the inequality (77) in the inequality (76) we obtain

$$
\begin{aligned}
\left\|\dot{y}_{\infty}\left(t, x_{\circ}, y_{\circ}\right)\right\| & \leq \frac{T}{2} R\left(N_{2}+J\right) M_{5} M_{11} \sigma_{\circ} R+\frac{T^{2}}{4} R^{2}\left(N_{2}+J\right) \delta_{1} M_{5} M_{11}\left(\frac{T}{2} R J M_{5}+1\right)+ \\
& +R \delta_{1} M_{5}
\end{aligned}
$$

by substitutions the two inequalities (77),(78) in the inequality (74) we obtain (66).

from the relation (63) we find that

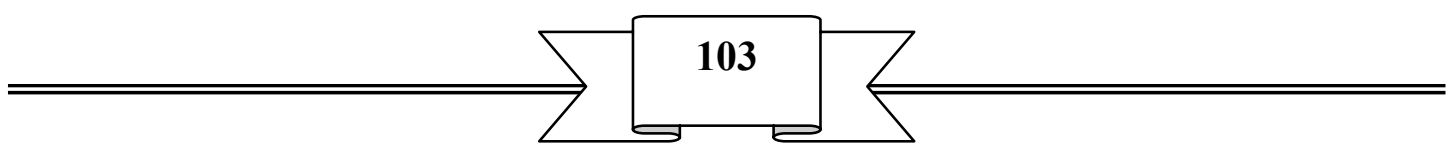




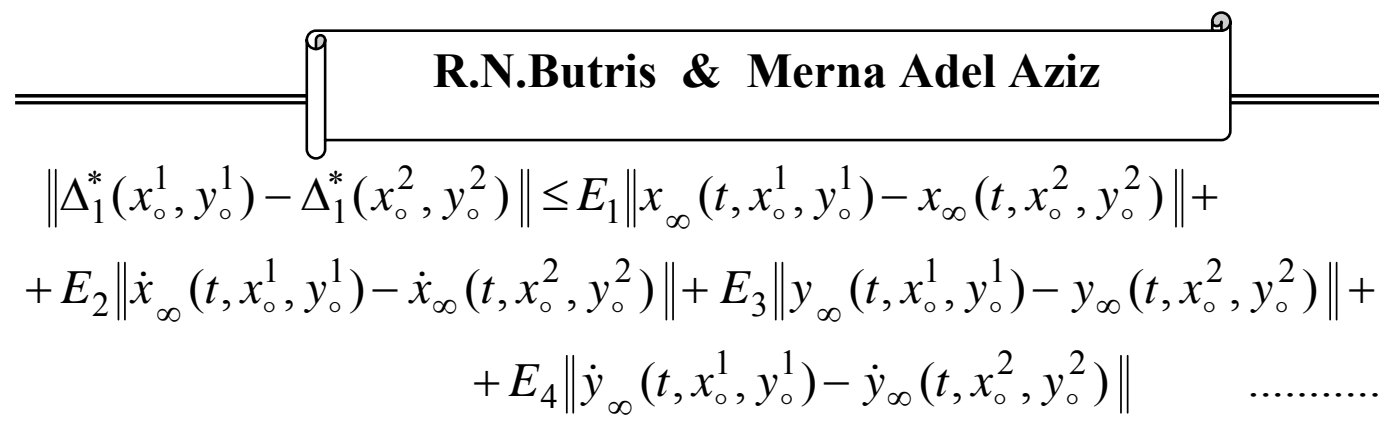

where

$x\left(t, x_{\circ}^{k}, y_{\circ}^{k}\right)=x_{\circ}^{k} e^{A t}+L^{2}\left(e^{A(t-s)} H\left(t, x_{\circ}^{k}, y_{\circ}^{k}\right)\right)$
$y\left(t, x_{\circ}^{k}, y_{\circ}^{k}\right)=y_{\circ}^{k} e^{C t}+L^{2}\left(e^{C(t-s)} Q\left(t, x_{\circ}^{k}, y_{\circ}^{k}\right)\right)$

where $\mathrm{k}=1,2$.

since $x_{\infty}\left(t, x_{\circ}, y_{\circ}\right), y_{\infty}\left(t, x_{\circ}, y_{\circ}\right)$ satisfies the two equations (28),(29) on arrangement .

from the relation (80) we find that

$$
\begin{gathered}
\left\|x_{\infty}\left(t, x_{\circ}^{1}, y_{\circ}^{1}\right)-x_{\infty}\left(t, x_{\circ}^{2}, y_{\circ}^{2}\right)\right\| \leq W_{1}\left\|x_{\circ}^{1}-x_{\circ}^{2}\right\| Q+\frac{T}{2} E_{2} W_{1}\left\|\dot{x}_{\infty}\left(t, x_{\circ}^{1}, y_{\circ}^{1}\right)-\dot{x}_{\infty}\left(t, x_{\circ}^{2}, y_{\circ}^{2}\right)\right\| \\
+\frac{T}{2} E_{3} W_{1}\left\|y_{\infty}\left(t, x_{\circ}^{1}, y_{\circ}^{1}\right)-y_{\infty}\left(t, x_{\circ}^{2}, y_{\circ}^{2}\right)\right\|+\frac{T}{2} E_{4} W_{1}\left\|\dot{y}_{\infty}\left(t, x_{\circ}^{1}, y_{\circ}^{1}\right)-\dot{y}_{\infty}\left(t, x_{\circ}^{2}, y_{\circ}^{2}\right)\right\| \ldots \ldots . .
\end{gathered}
$$

also we find that

$$
\begin{aligned}
& \left\|\dot{x}_{\infty}\left(t, x_{\circ}^{1}, y_{\circ}^{1}\right)-\dot{x}_{\infty}\left(t, x_{\circ}^{2}, y_{\circ}^{2}\right)\right\| \leq E_{1} W_{2}\left\|x_{\infty}\left(t, x_{\circ}^{1}, y_{\circ}^{1}\right)-x_{\infty}\left(t, x_{\circ}^{2}, y_{\circ}^{2}\right)\right\| \\
& +E_{3} W_{2}\left\|y_{\infty}\left(t, x_{\circ}^{1}, y_{\circ}^{1}\right)-y_{\infty}\left(t, x_{\circ}^{2}, y_{\circ}^{2}\right)\right\|+E_{4} W_{2}\left\|\dot{y}_{\infty}\left(t, x_{\circ}^{1}, y_{\circ}^{1}\right)-\dot{y}_{\infty}\left(t, x_{\circ}^{2}, y_{\circ}^{2}\right)\right\|
\end{aligned}
$$

from the relation $(81)$ we find that

$$
\begin{gathered}
\left\|y_{\infty}\left(t, x_{\circ}^{1}, y_{\circ}^{1}\right)-y_{\infty}\left(t, x_{\circ}^{2}, y_{\circ}^{2}\right)\right\| \leq W_{3}\left\|y_{\circ}^{1}-y_{\circ}^{2}\right\| R+\frac{T}{2} E_{5} W_{3}\left\|x_{\infty}\left(t, x_{\circ}^{1}, y_{\circ}^{1}\right)-x_{\infty}\left(t, x_{\circ}^{2}, y_{\circ}^{2}\right)\right\| \\
+\frac{T}{2} E_{6} W_{3}\left\|\dot{x}_{\infty}\left(t, x_{\circ}^{1}, y_{\circ}^{1}\right)-\dot{x}_{\infty}\left(t, x_{\circ}^{2}, y_{\circ}^{2}\right)\right\|+\frac{T}{2} E_{8} W_{3}\left\|\dot{y}_{\infty}\left(t, x_{\circ}^{1}, y_{\circ}^{1}\right)-\dot{y}_{\infty}\left(t, x_{\circ}^{2}, y_{\circ}^{2}\right)\right\|
\end{gathered}
$$

also we find that

$$
\begin{aligned}
& \left\|\dot{y}_{\infty}\left(t, x_{\circ}^{1}, y_{\circ}^{1}\right)-\dot{y}_{\infty}\left(t, x_{\circ}^{2}, y_{\circ}^{2}\right)\right\| \leq E_{5} W_{4}\left\|x_{\infty}\left(t, x_{\circ}^{1}, y_{\circ}^{1}\right)-x_{\infty}\left(t, x_{\circ}^{2}, y_{\circ}^{2}\right)\right\|+ \\
& +E_{6} W_{4}\left\|\dot{x}_{\infty}\left(t, x_{\circ}^{1}, y_{\circ}^{1}\right)-\dot{x}_{\infty}\left(t, x_{\circ}^{2}, y_{\circ}^{2}\right)\right\|+E_{7} W_{4}\left\|y_{\infty}\left(t, x_{\circ}^{1}, y_{\circ}^{1}\right)-y_{\infty}\left(t, x_{\circ}^{2}, y_{\circ}^{2}\right)\right\|
\end{aligned}
$$

after chain from substitutions in the inequalities (82),(83),(84),(85)we find 


\section{The existence and approximation ....}

$$
\begin{gathered}
\left\|x_{\infty}\left(t, x_{\circ}^{1}, y_{\circ}^{1}\right)-x_{\infty}\left(t, x_{\circ}^{2}, y_{\circ}^{2}\right)\right\| \leq\left[W_{1} W_{5} W_{8}+\frac{T}{2} E_{5} W_{1} W_{3}\left(W_{5}\right)^{2} W_{6} W_{8} W_{9} W_{10} W_{11} a_{1}+\right. \\
\left.+W_{1}\left(W_{5}\right)^{2}\left(W_{8}\right)^{2} W_{10} W_{11} a_{1} a_{7}\right]\left\|x_{\circ}^{1}-x_{\circ}^{2}\right\| Q+W_{3} W_{5} W_{6} W_{8} W_{9} W_{10} W_{11} a_{1}\left\|y_{\circ}^{1}-y_{\circ}^{2}\right\| R
\end{gathered}
$$

$\left\|y_{\infty}\left(t, x_{\circ}^{1}, y_{\circ}^{1}\right)-y_{\infty}\left(t, x_{\circ}^{2}, y_{\circ}^{2}\right)\right\| \leq\left[\frac{T}{2} E_{5} W_{1} W_{3} W_{5} W_{6} W_{9} W_{10} W_{11}+\right.$

$\left.+W_{1} W_{5} W_{8} W_{10} W_{11} a_{7}\right]\left\|x_{\circ}^{1}-x_{\circ}^{2}\right\| Q+W_{3} W_{6} W_{9} W_{10} W_{11}\left\|y_{\circ}^{1}-y_{\circ}^{2}\right\| R$

$\left\|\dot{y}_{\infty}\left(t, x_{\circ}^{1}, y_{\circ}^{1}\right)-\dot{y}_{\infty}\left(t, x_{\circ}^{2}, y_{\circ}^{2}\right)\right\| \leq\left[\frac{T}{2} E_{5} W_{1} W_{3}\left(W_{5}\right)^{2} W_{6} W_{7} W_{8} W_{9} W_{10} W_{11} a_{1} a_{4}+\right.$

$+W_{1}\left(W_{5}\right)^{2} W_{7}\left(W_{8}\right)^{2} W_{10} W_{11} a_{1} a_{4} a_{7}+W_{1} W_{5} W_{7} W_{8} a_{4}+$

$\left.+\frac{T}{2} E_{5} W_{1} W_{3} W_{5} W_{6} W_{7} W_{9} W_{10} W_{11} a_{5}+W_{1} W_{5} W_{7} W_{8} W_{10} W_{11} a_{5} a_{7}\right]\left\|x_{\circ}^{1}-x_{\circ}^{2}\right\| Q+$

$+\left[W_{3} W_{5} W_{6} W_{7} W_{8} W_{9} W_{10} W_{11} a_{1} a_{4}+W_{3} W_{6} W_{7} W_{9} W_{10} W_{11} a_{5}\right]\left\|y_{\circ}^{1}-y_{\circ}^{2}\right\| R$

$\left\|\dot{x}_{\infty}\left(t, x_{\circ}^{1}, y_{\circ}^{1}\right)-\dot{x}_{\infty}\left(t, x_{\circ}^{2}, y_{\circ}^{2}\right)\right\| \leq \frac{T}{2} E_{1} E_{5} W_{1} W_{2} W_{3}\left(W_{5}\right)^{2} W_{6} W_{8} W_{9} W_{10} W_{11} a_{1}+$

$+E_{1} W_{1} W_{2}\left(W_{5}\right)^{2}\left(W_{8}\right)^{2} W_{10} W_{11} a_{1} a_{7}+E_{1} W_{1} W_{2} W_{5} W_{8}+$

$+\frac{T}{2} E_{3} E_{5} W_{1} W_{2} W_{3} W_{5} W_{6} W_{9} W_{10} W_{11}+E_{3} W_{1} W_{2} W_{5} W_{8} W_{10} W_{11} a_{7}+$

$+\frac{T}{2} E_{4} E_{5} W_{1} W_{2} W_{3}\left(W_{5}\right)^{2} W_{6} W_{7} W_{8} W_{9} W_{10} W_{11} a_{1} a_{4}+$

$+E_{4} W_{1} W_{2}\left(W_{5}\right)^{2} W_{7}\left(W_{8}\right)^{2} W_{10} W_{11} a_{1} a_{4} a_{7}+E_{4} W_{1} W_{2} W_{5} W_{7} W_{8} a_{4}+$

$\left.+\frac{T}{2} E_{4} E_{5} W_{1} W_{2} W_{3} W_{5} W_{6} W_{7} W_{9} W_{10} W_{11} a_{5}+E_{4} W_{1} W_{2} W_{5} W_{7} W_{8} W_{10} W_{11} a_{5} a_{7}\right] \mid x_{\circ}^{1}-x_{\circ}^{2} \| Q+$

$+\left[E_{1} W_{2} W_{3} W_{5} W_{6} W_{8} W_{9} W_{10} W_{11} a_{1}+E_{3} W_{2} W_{3} W_{6} W_{9} W_{10} W_{11}+\right.$

$\left.+E_{4} W_{2} W_{3} W_{5} W_{6} W_{7} W_{8} W_{9} W_{10} W_{11} a_{1} a_{4}+E_{4} W_{2} W_{3} W_{6} W_{7} W_{9} W_{10} W_{11} a_{5}\right]\left\|y_{\circ}^{1}-y_{\circ}^{2}\right\| R$

by substitutions the inequalities (86),(87),(88),(89) in the inequality (79) we obtain (67).

also by the relation (64) we find that

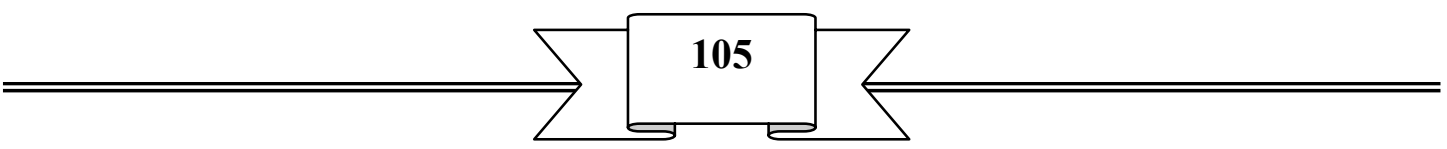




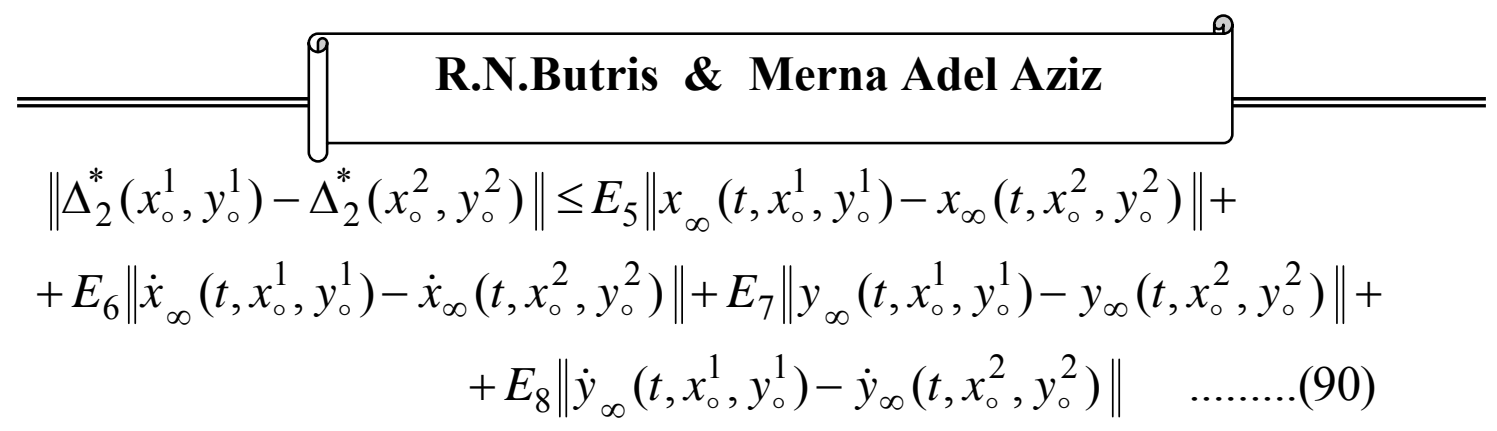

by substitutions the inequalities (86),(87),(88),(89) in the inequality (90) we obtain (68).

\section{Remark 2:-}

The theorem 4 confirm the stability of the solution for the system of non linear differential equations that is when a slight change happening in the point $\left(x_{\circ}, y_{\circ}\right)$ then a slight change will happen in the two functions $\Delta_{1}^{*}=\Delta_{1}^{*}\left(x_{\circ}, y_{\circ}\right), \Delta_{2}^{*}=\Delta_{2}^{*}\left(x_{\circ}, y_{\circ}\right)$

[for this remark return to [3] ].

\section{REFERENCES}

1- Butris, R. N. and AL -Ameen, M .S. The existence of periodic solutions for nonlinear systems of integro - differential equations, Iraq, Mosul,J. of Educ. And Sci Vol. 34, 37-48, (1999).

2- Martynuk, D. I. Periodic solutions of second - order nonlinear differential equation Ukrain, Math. J. No.4, pp. 125-132, (1967).

3- Mitropolsky, Yu. A. and Martynuk, D. I. Periodic solutions for the oscillations systems with retarded arguments, Ukrain, Kiev, (1979).

4- Naima, D. M. Periodic solutions of non autonomous second - order differential equations. boundary value problems - numerical solutionsdifferential equations, nonlinear studies, Vol. 6, Issue.8-14 ,(1999)

. 5- Perestyuk, N. A. The periodic solution for nonlinear systems of differential equation, Ukrain, Kiev, univ of kiev, Math. and Meca. J. No. 5, (1971).

6- Samoilenko, A . M. A numerical-analytic methods for investigations of periodic systems of ordinary differential equations I, II , Ukrain, Kiev, Math. J. No.4,5 , p.p. (82-93),(50-59),(1965,1966).

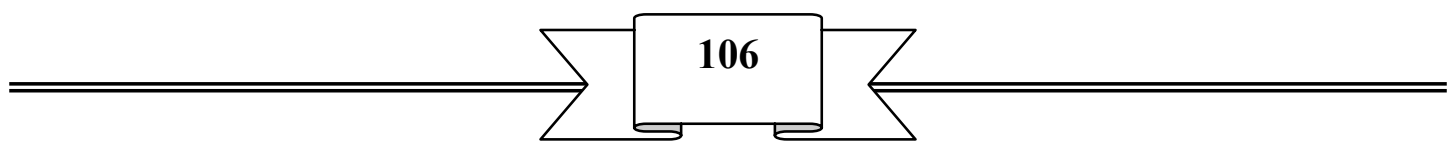

